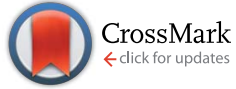

Cite this: RSC Adv., 2016, 6, 37347

Received 14th January 2016

Accepted 21st March 2016

DOI: $10.1039 / c 6 r a 01185 c$

www.rsc.org/advances

\section{Impact of strength and size of donors on the optoelectronic properties of $D-\pi-A$ sensitizers $\uparrow$}

\author{
J. Sivanadanam, ${ }^{\text {P }}$ P. Ganesan, ${ }^{\text {b Peng Gao, }}{ }^{\text {b }}$ Md. K. Nazeeruddin, ${ }^{\text {tb }}$ Alexei Emeline, ${ }^{\text {cd }}$ \\ Detlef Bahnemann ${ }^{\text {cd }}$ and R. Rajalingam ${ }^{\star a}$
}

A series of carbazole based sensitizers with either phenyl based donors (TBC, TMC, OMC, PC, TBR, TMR, OMR and PR) or aryl amine based donors (OMNC, CNC and HNC) as well as one without a donor group (CC) have been synthesized to understand the influence of the strength of the donor moiety on the optical, electrochemical and photovoltaic properties. Two different acceptor moieties such as cyano acrylic acid and rhodanine acetic acid were introduced and evaluated. Different substituents on the phenyl group have a significant impact on the light harvesting ability of the sensitizers. Among phenyl based donors, anisole based carbazole (OMC) shows the highest short circuit current $\left(J_{S C}\right)$ of $4.96 \mathrm{~mA}$ $\mathrm{cm}^{-2}$ with overall power conversion efficiency (PCE) of $2.69 \%$. In the case of the sensitizers with aryl amine based donors, the increasing bulkiness of the donor group lead to increasing open circuit potential. Transient photocurrent and photovoltage measurements signify the importance of a bulky donor fragment in determining the open circuit potential of the dyes. Sensitizers with hexyloxy substituted phenyl amine as the donor group shows a $J_{\text {SC }}$ of $6.84 \mathrm{~mA} \mathrm{~cm}{ }^{-2}$ with PCE of $3.33 \%$. The overall investigation provides vital information about the influence of donor groups on the optoelectronic properties of the sensitizers for its photovoltaic applications.

\section{Introduction}

Dye sensitized solar cells (DSSC) are one of the most promising methods to convert solar energy to electrical energy at low cost with high power conversion efficiency., ${ }^{\mathbf{1} 2}$ DSSC comprises four major components i.e., a semiconductor metal oxide, sensitizer, redox active electrolyte and counter electrode. Sensitizers play a major role in harvesting visible light radiations to achieve high power conversion efficiency and have been intensively studied. To date, dyes based on ruthenium complexes and porphyrin sensitizers achieved maximum power conversion efficiencies of 11 and 13\%, respectively. ${ }^{1,3}$ Development of metal free organic sensitizers has been increased vastly in this decade due to the rare, expensive ruthenium metal and the difficulties in purification process. Organic sensitizers however have

${ }^{a}$ School of Chemistry, Bharathidasan University, Tiruchirappalli 620024, Tamilnadu, India..E-mail: rrengas@gmail.com

${ }^{b}$ Group for Molecular Engineering of Functional Materials, EPFL Valais Wallis, Rue de l'industrie 17, CH-1951 Sion, Switzerland. E-mail: peng.gao@epfl.ch; mdkhaja. nazeeruddin@epfl.ch

"Laboratory "Photoactive Nanocomposite Materials", Saint-Petersburg State University, Ulyanovskaya str. 1 Peterhof, Saint-Petersburg, 198504 Russia. E-mail: detlef.bahnemann@spbu.ru

"Photocatalysis and Nanotechnology", Institut fuer Technische Chemie, Gottfried Wilhelm Leibniz Universitaet Hannover, Callinstrasse 3, D-30167 Hannover, Germany.E-mail: bahnemann@iftc.uni-hannover.de

$\dagger$ Electronic supplementary information (ESI) available: Synthetic procedure of carbazole intermediates and the characterization. See DOI: 10.1039/c6ra01185c advantages like high molar extinction coefficient, structural flexibility, low cost, easier preparation and purification process. In the past years, sensitizers with various aromatic building block such as coumarin, ${ }^{4}$ indoline ${ }^{5}$ perylene $^{6}{ }^{6}$ merocyanine, ${ }^{7}$ porphyrin, $^{8}$ triarylamine ${ }^{9}$ and carbazole ${ }^{10}$ have been reported, all of which have obtained power conversion efficiency in the range of $5-9 \%$.

Carbazole is a well-known heterocyclic compound which has unique optoelectronic properties, and has found applications in hole transporting material in organic devices, electron donors in solar cells and host material in electroluminescent devices. ${ }^{\mathbf{1 1 , 1 2}}$ Chemically, carbazole is easily functionalized at 3,6 and 9 positions and covalently bonded to other molecular compounds. ${ }^{13,14}$ Recently, Jianhua $\mathrm{Su}^{14}$ reported the $\mathrm{D}-\pi-\mathrm{A}$ molecules functionalized at the above positions showing the efficiency up to $5.91 \%$. Most recently, sensitizer (MK-2) based on carbazole reported by Z. S. Wang and co-workers produced a maximum power conversion efficiency of $8.3 \% .{ }^{13}$ The classic structural arrangement for organic dyes comprises the donor fragment and acceptor fragment linked by the $\pi$ spacer (D- $\pi-$ A). Among various types of donor groups utilized in the DSSC, aryl amine based donor groups have been most popular options achieving reasonable efficiencies. ${ }^{15}$ Increasing the twist angle between the donor and $\pi$ spacer will avoid the dye aggregation that eventually assists in reducing the charge recombination between the injected electrons and the oxidized dyes. Recently, fluorenes and dibenzo heterocycles such as carbazole were 


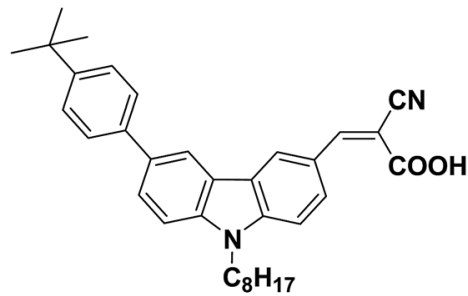

TBC

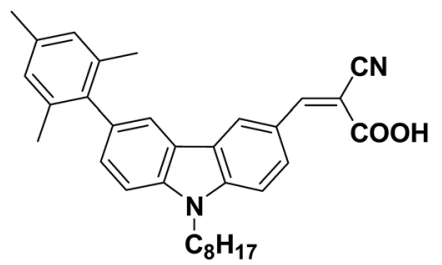

TMC

$\mathrm{MeO}$<smiles>CCCCn1c2ccc(/C=C(/C#N)C(=O)O)cc2c2cc(-c3ccc(Cl)cc3)ccc21</smiles>

OMC

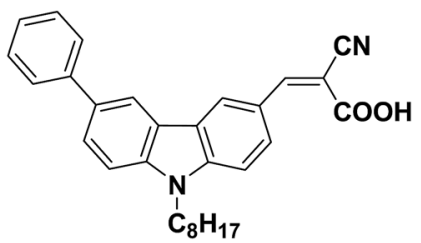

PC<smiles>[R7][R7](Cn1c2ccc(/C=C3\SC(=S)N(CC(=O)O)C3=O)cc2c2cc(-c3ccc(C(C)(C)C)cc3)ccc21)[I-][3H]</smiles><smiles>Cc1cc(C)c(-c2ccc3c(c2)c2cc(/C=C4\SC(=S)N(CC(=O)O)C4=O)ccc2n3Cc2ccccc2)c(C)c1</smiles>

TMR

$\mathrm{MeO}$

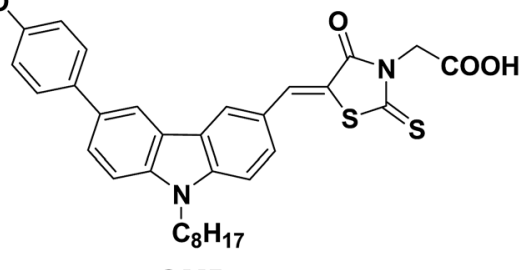

OMR

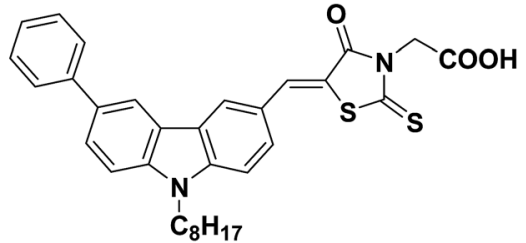

PR

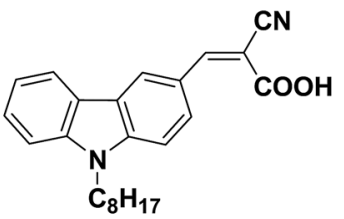

CC

Scheme 1 Structures of carbazole sensitizers with phenyl based donors.

successfully employed as $\pi$ spacer by gratifying the above mentioned criteria. ${ }^{\mathbf{1 6}}$ Further functionalization of different donor moieties in the carbazole fragment is easy and convenient that will aid in constructing the required donor- $\pi-$ acceptor structural arrangement. In this context, a complete study on the impact of strength of donor groups in a simple D$\pi-\mathrm{A}$ sensitizers will unveil the role of the donor group in the light harvesting ability, electrochemical and photovoltaic parameters.

Based on these points, in this paper we have synthesised carbazole based sensitizers with/without donor groups of different donating strength. All the new sensitizers were successfully synthesised and characterized. The differences in the strength of the donor fragments reflected in the absorption, emission, electrochemical properties of the carbazole sensitizers. The variations in the photovoltaic parameters such as short circuit current density $J_{\mathrm{SC}}$, open circuit potential $V_{\mathrm{OC}}$ by varying the substituents in the donor fragments were discussed. The potential of the sensitizers to convert the absorbed light in to current was analysed using IPCE measurements. Transient photocurrent and photovoltage measurements were carried out to analyse the importance of bulky donor fragment in determining the open circuit potential of the dyes.

\section{Experimental part}

Carbazole, 1-bromo octane, rhodanine acetic acid, (2,4,6-trimethylphenyl)boronic acid, 4-methoxyphenylboronic acid, 4phenylboronic acid and $\mathrm{Pd}\left(\mathrm{PPh}_{3}\right)_{4}$ were purchased from Aldrich. 1,2-Dichloroethane, sodium hydroxide, potassium iodide, potassium carbonate and 100-200 mesh silica gel were from Merck chemicals. Phosphorous oxy chloride, cyano acetic 
<smiles>CCCn1c2ccc(C=C(C#N)C(=O)O)cc2c2cc(N(c3ccc(OC)cc3)c3ccc(OC)cc3)ccc21</smiles>

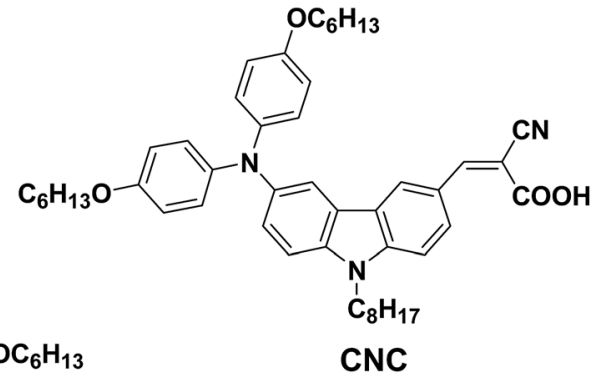

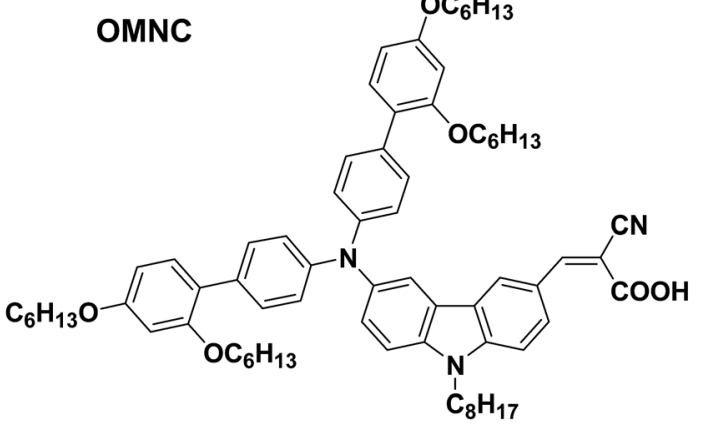

HNC

Scheme 2 Structures of carbazole sensitizers with amine based donors.

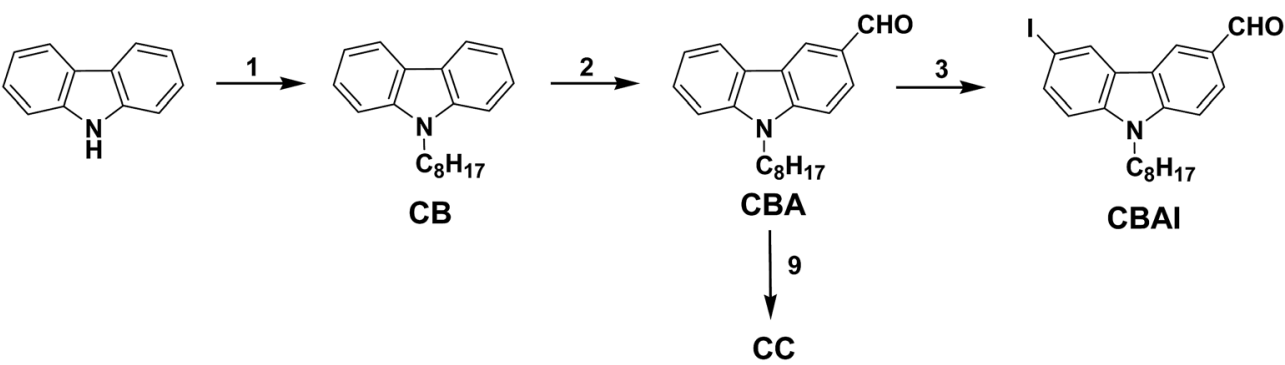

Scheme 3 Synthetic pathways for the preparation of CBAl. Reagents: (1) carbazole, 1-bromo octane, NaOH, DMSO, RT. (2) CB, DMF, POCl 3 , $\mathrm{DCE}, 90^{\circ} \mathrm{C}$. (3) $\mathrm{CBA}, \mathrm{KI}, \mathrm{KIO}_{3}$, acetic acid, $80^{\circ} \mathrm{C}$.

acid and ammonium acetate were obtained from Loba chemicals. 4-tert-Butylphenylboronic acid and potassium iodate from Spectrochem. All solvents were purchased from spectrometric grade and used as received without further purification. ${ }^{1} \mathrm{H}$ NMR and ${ }^{13} \mathrm{C}$ NMR spectrum was recorded on a Bruker 400 $\mathrm{MHz}$ spectrometer in $\mathrm{CDCl}_{3}-d$ or DMSO- $d_{6}$ solvent with tetramethylsilane as internal standard. Mass spectra were obtained with Bruker daltonics, FT-ICR/APEX II, ESI mode. MALDI-TOF mass spectra were recorded using Micromass Tof Spec 2E instrument. High-resolution mass spectra were obtained at the École Polytechnique Fédérale de Lausanne mass spectrometry laboratory (EPFL).

Absorption spectra of dyes in chloroform solution and on $\mathrm{TiO}_{2}$ films were recorded using a JASCO $300 \mathrm{UV}$-Visible spectrometer. Fluorescence measurements were carried out using Perkin Elmer LS 55 spectrofluorimeter with $10 \mathrm{~nm}$ slit width and $200 \mathrm{~nm} \min ^{-1}$ scan width was kept constant for all measurements. Electrochemical studies were examined by cyclic voltammetry (SP-50 model potentiostat) using a three electrode system. The working electrode was a platinum electrode, the counter electrode was a platinum wire and the reference electrode was a $\mathrm{Ag} / \mathrm{AgCl}$. Tetrabutylammonium hexafluoro phosphate $\left(\mathrm{TBAPF}_{6}, 0.1 \mathrm{M}\right)$ was used as supporting electrolyte in acetonitrile solution for phenyl based donors and in dichloromethane solutions for amine based donors. A pinch of ferrocene was added to each sample and ferrocene/ ferrocenium $\left(\mathrm{Fc} / \mathrm{Fc}^{+}\right)$was used as the internal standard. The potential of the dyes $v s$. normal hydrogen electrode (NHE) were calibrated by using ferrocene $(0.630 \mathrm{~V} v s$. NHE) as the internal standard. ${ }^{17}$ All calculations were carried out using Spartan'10 Windows run on Microsoft Windows XP, Geometry optimization, energy levels, and frontier molecular orbitals of the dyes' HOMOs and LUMOs were calculated at the B3LYP/6-31G $(\mathrm{d}, \mathrm{p})$ level.

A $450 \mathrm{~W}$ xenon lamp (Oriel, USA) was used as a light source to study the current-voltage characteristics of the DSSC. The spectral output of the lamp was filtered using a Schott K113 Tempax sunlight filter (Präzisions Glas \& Optik GmbH, Germany) to reduce the mismatch between the simulated and actual solar spectrum to less than $2 \%$. The Keithley model 2400 


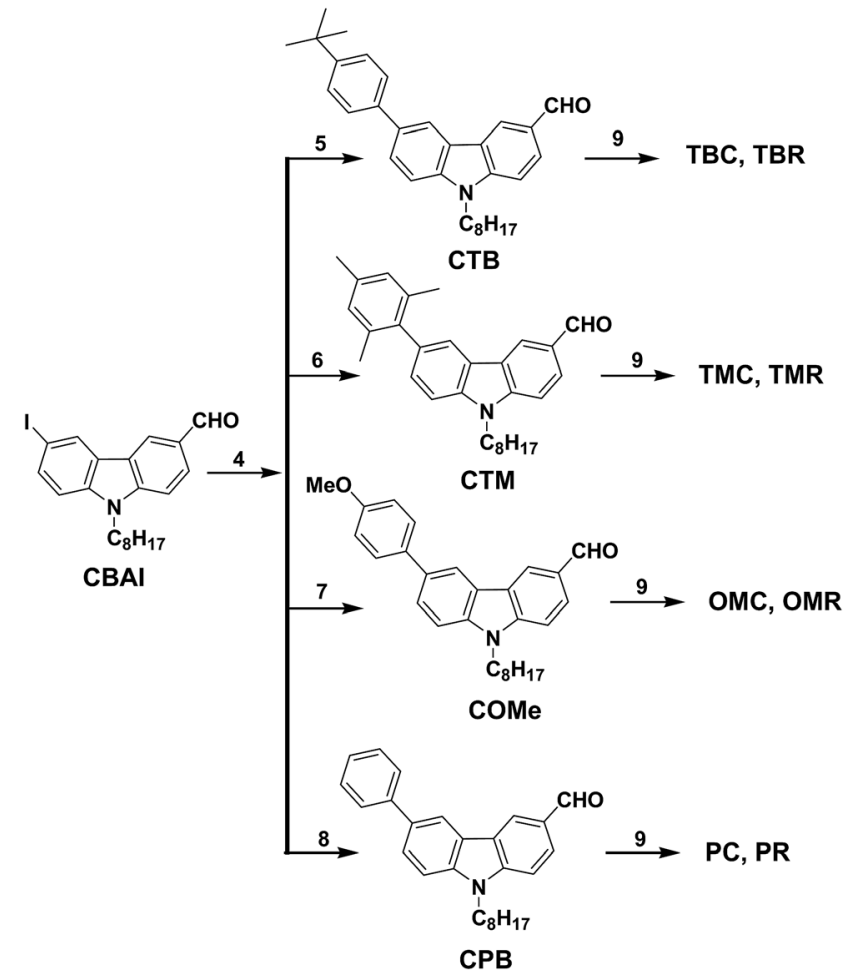

Scheme 4 Synthetic routes for the preparation of phenyl donor based carbazole sensitizers. Reagents: (4) CBAl, (4-tert-butylphenylboronic acid (5) 2,4,6-trimethylphenyl)boronic acid (6), 4-methoxyphenylboronic acid (7), 4-phenylboronic acid (8), $\mathrm{Pd}\left(\mathrm{PPh}_{3}\right)_{4}, \mathrm{THF}$, $\mathrm{H}_{2} \mathrm{O}, 70^{\circ} \mathrm{C}$. (9) $\mathrm{CBA}, \mathrm{CTB}, \mathrm{CTM}, \mathrm{COMe}$ and $\mathrm{CPB}$, ammonium acetate, acetic acid, $120^{\circ} \mathrm{C}$

digital source meter (Keithley, USA) was used for data acquisition. The photo-active area of $0.16 \mathrm{~cm}^{2}$ was defined by a black mask of $6 \times 6 \mathrm{~mm}^{2}$. Incident photon-to-current conversion efficiency measurements were measured using the mono chromated visible photons, from Gemini-180 double monochromator Jobin Yvon Ltd (UK), powered by a $300 \mathrm{~W}$ xenon light source (ILC Technology, USA) superimposed on a $10 \mathrm{~mW} \mathrm{~cm}^{-2}$ LED light. The monochromatic incident light was passed through a chopper running at $2 \mathrm{~Hz}$ frequency and the on/off ratio was measured by an operational amplifier. Photovoltage transients were observed by using a pump pulse generated by 4 red light emitting diodes controlled by a fast solid-state switch with a white light bias. The pulse of red light with widths of 50 ms was incident on the photoanode side of the cell, and its intensity was controlled to keep a suitably low level to generate the exponential voltage decay where the charge recombination rate constants are obtained directly from the exponential decay rate. $^{18}$

\section{Device fabrication}

A screen-printed double layer of nanocrystalline $\mathrm{TiO}_{2}$ particles was used as the photoelectrode. The FTO glass plates were immersed in a $40 \mathrm{mM}$ aqueous $\mathrm{TiCl}_{4}$ solution at $70{ }^{\circ} \mathrm{C}$ for 30 min and washed with water and ethanol. A $8 \mu \mathrm{m}$ thick film of 20 nm sized $\mathrm{TiO}_{2}$ particles was then printed on the FTO conducting glass and further coated with a $5 \mu \mathrm{m}$ thick second layer of light-scattering $\mathrm{TiO}_{2}$ particles (400 nm diameter, Catalysts \& Chemicals Ind. Co. Ltd (CCIC), HPW-400). Sintering was carried out at $500{ }^{\circ} \mathrm{C}$ for $15 \mathrm{~min}$, which was gradually heated. The working electrode was prepared by immersing the $13.0 \mu \mathrm{m}(8.0$ $\mu \mathrm{m}$ thick transparent layer $+5.0 \mu \mathrm{m}$ thick scattering) $\mathrm{TiO}_{2}$ film into the dye solution for $12 \mathrm{~h}$. To prepare the counter electrode, Pt catalyst was deposited on cleaned FTO glass by coating with a drop of $\mathrm{H}_{2} \mathrm{PtCl}_{6}$ solution (10 mM in 2-propanol solution) with heat treatment at $400{ }^{\circ} \mathrm{C}$ for $15 \mathrm{~min}$. For the assembly of DSSCs, the dye-containing $\mathrm{TiO}_{2}$ electrode and Pt counter electrode were assembled into a sandwich-type cell and sealed with a hot-melt gasket of 25 microns thickness made of the ionomer Surlyn 1702 (Dupont). Devices were completed by filling the electrolyte through the pre-drilled holes in the counter electrodes and finally the holes were sealed with a Surlyn sheet and a thin glass cover by heating. A black mask $(6 \times 6 \mathrm{~mm})$ was used in the subsequent photovoltaic studies.

\section{Synthesis and characterization of carbazole based sensitizers}

The complete synthetic procedures of all intermediate compounds are available in ESI. $\dagger$ Three amine donor moieties $(11,12$ and 13$)$ are synthesized according to the literature. ${ }^{19,20}$

\subsection{General procedure for the preparation of target compounds}

To a mixture of corresponding aldehydes (1 mol ratio), cyanoacetic acid or rhodanine acetic acid (1.5 mol ratio) and ammonium acetate $(1.95 \mathrm{~mol}$ ratio) in glacial acetic acid $(5 \mathrm{ml})$ was stirred at $80^{\circ} \mathrm{C}$ for 1 day. After completion of the reaction, cool to RT followed by quench the mixture in ice water, a precipitate was obtained. It was filtered and dried to afford the desired product.

3-[6-(4-tert-Butyl-phenyl)-9-octyl-9H-carbazol-3-yl]-2-cyanoacrylic acid (TBC). Product: yellow colored solid, yield: $71 \%$. Melting point: $244{ }^{\circ} \mathrm{C}^{1} \mathrm{H} \mathrm{NMR},\left(400 \mathrm{MHz}, \mathrm{CDCl}_{3}-d\right): \delta=8.78$ $(\mathrm{s}, 1 \mathrm{H}), 8.48(\mathrm{~s}, 1 \mathrm{H}), 8.32(\mathrm{~s}, 1 \mathrm{H}), 8.26(\mathrm{~d}, J=8 \mathrm{~Hz}, 1 \mathrm{H}), 7.77(\mathrm{~d}$, $J=8.4 \mathrm{~Hz}, 1 \mathrm{H}), 7.64(\mathrm{~d}, J=8 \mathrm{~Hz}, 2 \mathrm{H}), 7.52-7.46(\mathrm{~m}, 4 \mathrm{H}), 4.31$ $(\mathrm{s}, 2 \mathrm{H}), 1.89(\mathrm{~s}, 2 \mathrm{H}), 1.39(\mathrm{~s}, 9 \mathrm{H}), 1.33-1.25(\mathrm{~m}, 10 \mathrm{H}), 0.86(\mathrm{t}, J$ $=6.4 \mathrm{~Hz}, 3 \mathrm{H}) \mathrm{ppm} .{ }^{13} \mathrm{C} \mathrm{NMR},\left(100 \mathrm{MHz}, \mathrm{CDCl}_{3}-d\right): \delta=168.77$, 157.21, 149.88, 143.91, 140.32, 138.42, 134.11, 129.58, 126.95, 126.30, 125.84, 123.66, 123.24, 122.47, 119.11, 116.77, 109.63, 109.50, 43.51, 34.54, 31.76, 31.43, 29.72, 29.29, 29.15, 28.95, $27.24,22.60,14.06 \mathrm{ppm}$. HRMS (ESI-TOF) $\mathrm{m} / \mathrm{z}$ : calculated for $\mathrm{C}_{34} \mathrm{H}_{38} \mathrm{~N}_{2} \mathrm{O}_{2}[\mathrm{M}+\mathrm{H}]^{+}$: 506.29, found: 507.3193.

\{5-[6-(4-tert-Butyl-phenyl)-9-octyl-9H-carbazol-3-ylmethylene]4-oxo-2-thioxo-thiazolidin-3-yl\}-acetic acid (TBR). Product: orange colored solid, yield: $72 \%$. Melting point: $254{ }^{\circ} \mathrm{C}{ }^{1} \mathrm{H}$ NMR, (400 MHz, DMSO- $\left.d_{6}\right): \delta=8.53-8.47(\mathrm{~m}, 2 \mathrm{H}), 7.98(\mathrm{~s}, 1 \mathrm{H}), 7.74-$ $7.65(\mathrm{~m}, 6 \mathrm{H}), 7.45(\mathrm{~d}, J=8.4 \mathrm{~Hz}, 2 \mathrm{H}), 4.69(\mathrm{~s}, 2 \mathrm{H}), 4.39(\mathrm{~s}, 2 \mathrm{H})$, $1.73(\mathrm{~s}, 2 \mathrm{H}), 1.28(\mathrm{~s}, 9 \mathrm{H}), 1.20-1.10(\mathrm{~m}, 10 \mathrm{H}), 0.74(\mathrm{t}, J=4.8 \mathrm{~Hz}$, $3 \mathrm{H}$ ) ppm. HRMS (ESI-TOF) $m / z$ : calculated for $\mathrm{C}_{36} \mathrm{H}_{40} \mathrm{~N}_{2} \mathrm{O}_{3} \mathrm{~S}_{2}[\mathrm{M}+$ $\mathrm{H}]^{+}: 612.25$, found: 613.2781 . 


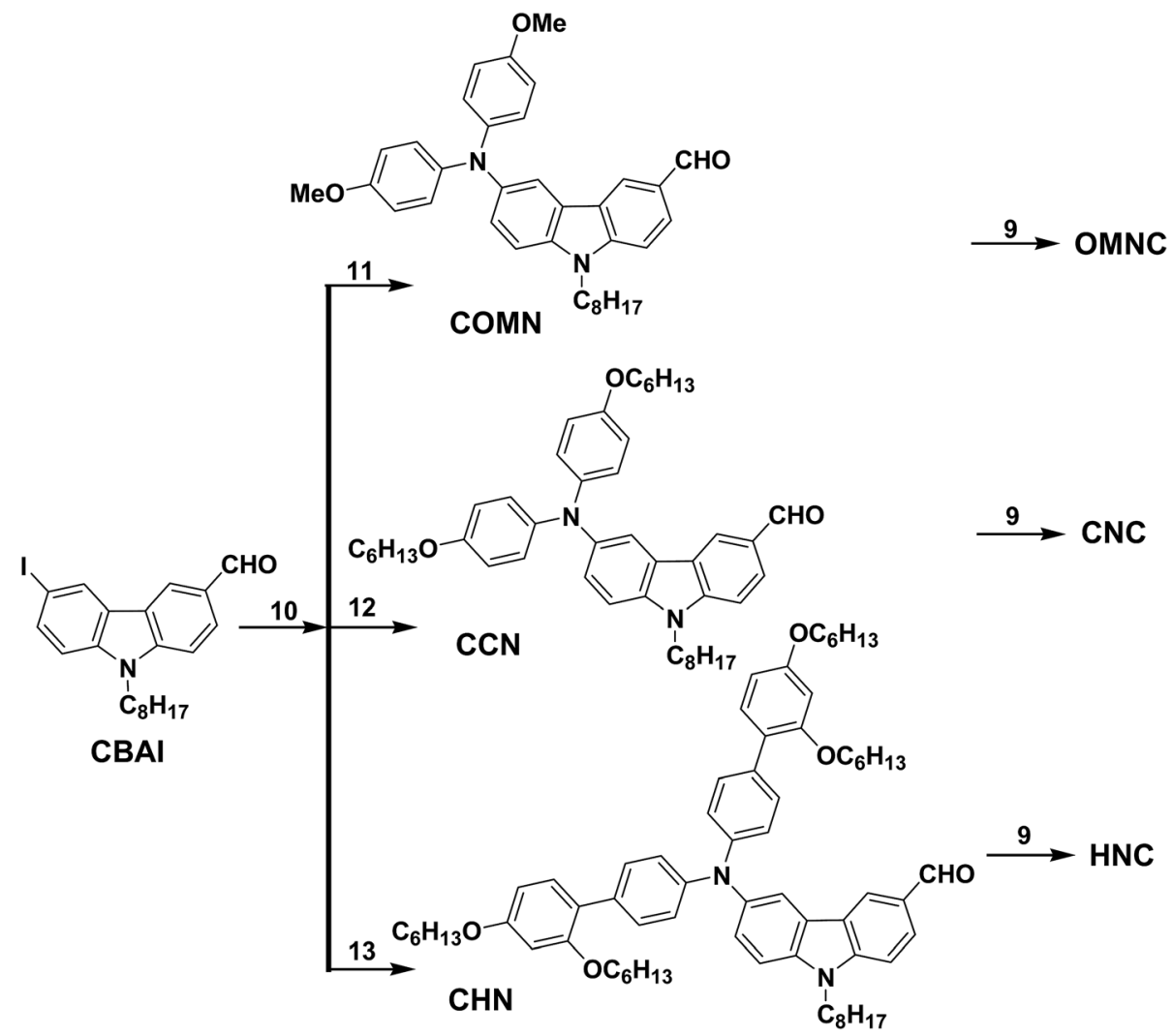

Scheme 5 Synthetic routes for the preparation of amine donor based carbazole sensitizers. Reagents: (10) CBAI, bis-(4-methoxy-phenyl)-amine (11), bis-(4-hexyloxy-phenyl)-amine (12), bis(2',4'-bis(hexyloxy)-[1,1'-biphenyl]-4-yl)amine (13), Pd[P(t-Bu) $]_{2}$, sodium tert-butoxide, toluene, 80 ${ }^{\circ} \mathrm{C}$. (9) $\mathrm{COMN}, \mathrm{CCN}$ and $\mathrm{CHN}$, ammonium acetate, acetic acid, $120{ }^{\circ} \mathrm{C}$.

2-Cyano-3-[9-octyl-6-(2,4,6-trimethyl-phenyl)-9H-carbazol-3-yl]acrylic acid (TMC). Product: yellow colored solid, yield: $57 \%$. Melting point: $226{ }^{\circ} \mathrm{C}^{1} \mathrm{H}$ NMR, $\left(400 \mathrm{MHz}, \mathrm{CDCl}_{3}-d\right): \delta=8.75$ (s, $1 \mathrm{H}), 8.46(\mathrm{~s}, 1 \mathrm{H}), 8.26(\mathrm{~d}, J=8.8 \mathrm{~Hz}, 1 \mathrm{H}), 7.91(\mathrm{~s}, 1 \mathrm{H}), 7.49(\mathrm{~d}, J=$ $7.6 \mathrm{~Hz}, 2 \mathrm{H}), 7.31(\mathrm{~d}, J=8.3 \mathrm{~Hz}, 1 \mathrm{H}), 6.99(\mathrm{~s}, 2 \mathrm{H}), 4.36(\mathrm{t}, J=7.2$ $\mathrm{Hz}, 2 \mathrm{H}), 2.36(\mathrm{~s}, 3 \mathrm{H}), 2.03(\mathrm{~s}, 6 \mathrm{H}), 1.95(\mathrm{t}, J=7.2 \mathrm{~Hz}, 2 \mathrm{H}), 1.44-$ $1.25(\mathrm{~m}, 10 \mathrm{H}), 0.87(\mathrm{t}, J=6 \mathrm{~Hz}, 3 \mathrm{H}) \mathrm{ppm} .{ }^{13} \mathrm{C} \mathrm{NMR},(100 \mathrm{MHz}$, $\mathrm{CDCl}_{3}-d$ ): $\delta=168.99,157.52,144.02,140.01,138.96,136.71$, 136.48, 133.88, 129.60, 128.57, 128.15, 126.04, 123.71, 122.97,
122.34, 121.47, 116.58, 109.60, 109.52, 96.36, 43.70, 31.79, 29.34, 29.19, 29.06, 27.34, 22.63, 21.10, 21.01, 14.10 ppm. HRMS (ESITOF) $m / z$ : calculated for $\mathrm{C}_{33} \mathrm{H}_{36} \mathrm{~N}_{2} \mathrm{O}_{2}[\mathrm{M}+\mathrm{H}]^{+}: 492.28$, found: 493.2904.

\{5-[9-Octyl-6-(2,4,6-trimethyl-phenyl)-9H-carbazol-3-ylmethylene]-4-oxo-2-thioxo-thiazolidin-3-yl\}-acetic acid (TMR). Product: orange colored solid, yield: $72 \%$. Melting point: $214{ }^{\circ} \mathrm{C}{ }^{1} \mathrm{H}$ NMR, $\left(400 \mathrm{MHz}, \mathrm{CDCl}_{3}-d\right): \delta=8.21(\mathrm{~s}, 1 \mathrm{H}), 8.00(\mathrm{~s}, 1 \mathrm{H}), 7.89(\mathrm{~s}, 1 \mathrm{H})$, $7.62(\mathrm{~d}, J=8 \mathrm{~Hz}, 1 \mathrm{H}), 7.48(\mathrm{~d}, J=8.8 \mathrm{~Hz}, 2 \mathrm{H}), 7.31(\mathrm{~d}, J=8 \mathrm{~Hz}$,
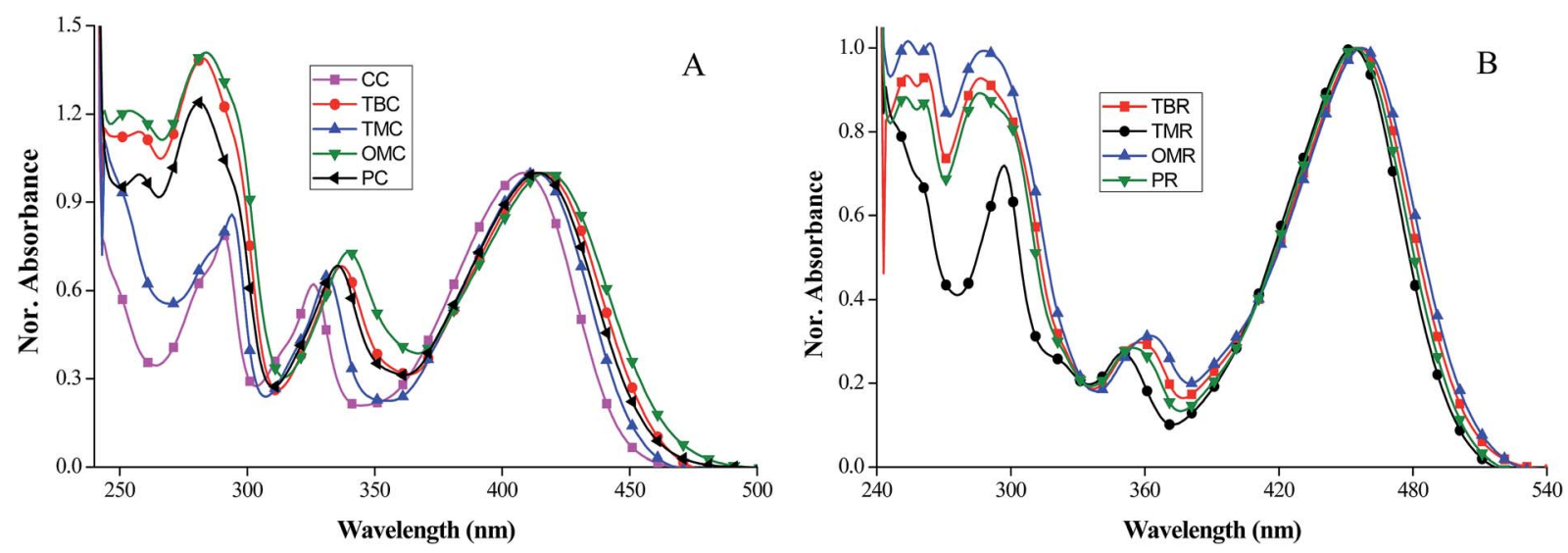

Fig. 1 Normalised absorption spectrum of phenyl donor based carbazole dyes in $\mathrm{CHCl}_{3}$ solutions. 
Table 1 Absorption, emission and electrochemical properties of phenyl donor based carbazole dyes

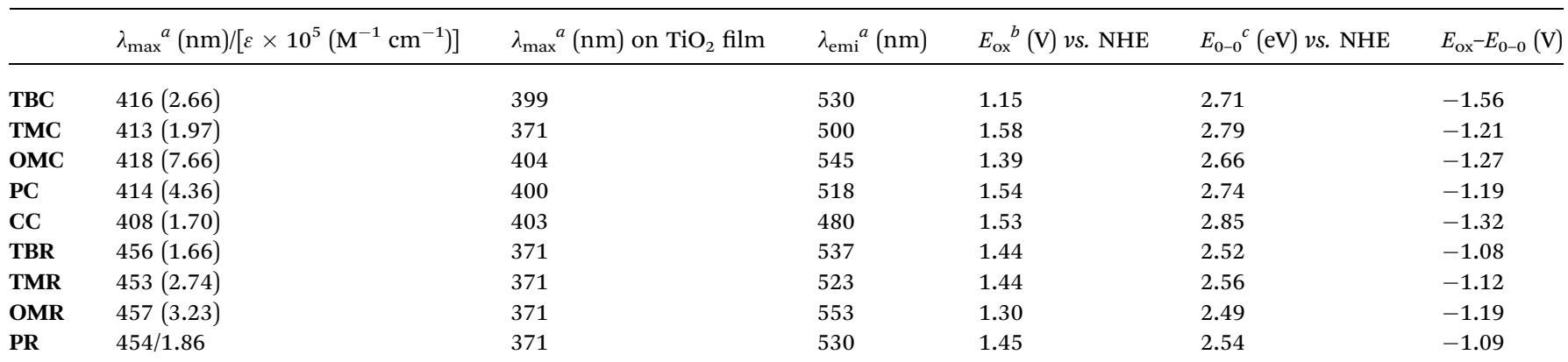

${ }^{a}$ Absorption and emission spectrum of phenyl donor based carbazole dyes were measured in $\mathrm{CHCl}_{3}$ solutions. ${ }^{b}$ Cyclic voltammograms of the first oxidation potential of the dyes were measured in acetonitrile solution containing $0.1 \mathrm{M} \mathrm{TBAPF}_{6}$ used as an supporting electrolyte with a scan rate of $100 \mathrm{mV} \mathrm{s}^{-1}$ (working electrode: Pt, counter electrode: $\mathrm{Pt}$ wire, reference electrode: $\mathrm{Ag} / \mathrm{AgCl}$ calibrated with ferrocene/ferrocenium $\left(\mathrm{Fc} / \mathrm{Fc}^{+}\right.$) as an internal reference and converted to NHE by adding $630 \mathrm{mV}$ ). ${ }^{c}$ The $E_{0-0}$ transition values was estimated from the onset of absorption and emission spectra.

1H), $6.99(\mathrm{~s}, 2 \mathrm{H}), 4.94(\mathrm{~s}, 2 \mathrm{H}), 4.34(\mathrm{t}, J=7.2 \mathrm{~Hz}, 2 \mathrm{H}), 2.37(\mathrm{~s}, 3 \mathrm{H})$, $2.04(\mathrm{~s}, 6 \mathrm{H}), 1.93$ (p, $J=7.2 \mathrm{~Hz}, 2 \mathrm{H}), 1.47-1.25(\mathrm{~m}, 10 \mathrm{H}), 0.87(\mathrm{t}, J$ $=6 \mathrm{~Hz}, 3 \mathrm{H}) \mathrm{ppm} .{ }^{13} \mathrm{C} \mathrm{NMR},\left(100 \mathrm{MHz} \mathrm{CDCl}_{3}-d\right): \delta=192.97$, 171.36, 167.30, 142.13, 139.94, 139.08, 136.68, 136.52, 136.31, $133.33,128.93,128.34,128.15,124.29,124.04,123.88,122.75$, 121.28, 117.94, 109.74, 109.34, 44.41, 43.60, 31.79, 29.73, 29.35, 29.19, 29.07, 27.35, 22.63, 21.10, 21.03, 14.10 ppm. HRMS (ESITOF) $m / z$ : calculated for $\mathrm{C}_{35} \mathrm{H}_{38} \mathrm{~N}_{2} \mathrm{O}_{3} \mathrm{~S}_{2}[\mathrm{M}+\mathrm{H}]^{+}$: 598.23 , found: 599.2596 .

2-Cyano-3-[6-(4-methoxy-phenyl)-9-octyl-9H-carbazol-3-yl]-acrylic acid (OMC). Product: dark yellow colored solid, yield: 93\%. Melting point: $182{ }^{\circ} \mathrm{C}^{1} \mathrm{H}$ NMR, (400 MHz, DMSO- $\left.d_{6}\right): \delta=8.83(\mathrm{~s}, 1 \mathrm{H}), 8.37$ $(\mathrm{s}, 1 \mathrm{H}), 8.32(\mathrm{~s}, 1 \mathrm{H}), 8.25(\mathrm{dd}, J=8.8,1.6 \mathrm{~Hz}, 1 \mathrm{H}), 7.77-7.62(\mathrm{~m}, 5 \mathrm{H})$, $7.00(\mathrm{~d}, J=8.8 \mathrm{~Hz}, 2 \mathrm{H}), 4.40(\mathrm{t}, J=6.4 \mathrm{~Hz}, 2 \mathrm{H}), 3.75(\mathrm{~s}, 3 \mathrm{H}), 1.74(\mathrm{t}, J$ $=6.4 \mathrm{~Hz}, 2 \mathrm{H}), 1.20-1.11(\mathrm{~m}, 10 \mathrm{H}), 0.75(\mathrm{t}, J=8.4 \mathrm{~Hz}, 3 \mathrm{H}) \mathrm{ppm} .{ }^{13} \mathrm{C}$ NMR, (100 MHz, DMSO- $\left.d_{6}\right): \delta=164.14,158.43,155.07,143.03$, $139.73,133.05,132.53,127.74,127.51,125.99,125.49,122.61$, $122.28,117.93,117.25,114.33,110.45,110.31,98.28,55.09,42.63$, 31.10, 28.63, 28.53, 28.46, 26.31, 21.95, $13.82 \mathrm{ppm}$. HRMS (ESI) $\mathrm{m} / z$ : calculated for $\mathrm{C}_{31} \mathrm{H}_{32} \mathrm{~N}_{2} \mathrm{O}_{3}[\mathrm{M}-\mathrm{H}]^{+}$: 480.24 , found: 479.42 .

\{5-[6-(4-Methoxy-phenyl)-9-octyl-9H-carbazol-3-ylmethylene]4-oxo-2-thioxo-thiazolidin-3-yl\}-acetic acid (OMR). Product: brick red colored solid, yield: $92 \%$. Melting point: $110{ }^{\circ} \mathrm{C}{ }^{1} \mathrm{H}$ NMR, $\left(400 \mathrm{MHz}\right.$, DMSO- $\left.d_{6}\right): \delta=8.48(\mathrm{~d}, J=10.8 \mathrm{~Hz}, 2 \mathrm{H}), 7.96$ (s, $1 \mathrm{H}), 7.73-7.63(\mathrm{~m}, 6 \mathrm{H}), 7.00(\mathrm{~d}, J=8.8 \mathrm{~Hz}, 2 \mathrm{H}), 4.66(\mathrm{~s}, 2 \mathrm{H}), 4.38$ (s, 2H), $3.75(\mathrm{~s}, 3 \mathrm{H}), 1.73-1.71(\mathrm{~m}, 2 \mathrm{H}), 1.20-1.11(\mathrm{~m}, 10 \mathrm{H}), 0.74$ $(\mathrm{t}, J=6.8 \mathrm{~Hz}, 3 \mathrm{H})$ ppm. MS (ESI) $m / z$ : calculated for $\mathrm{C}_{33} \mathrm{H}_{34} \mathrm{~N}_{2} \mathrm{O}_{4} \mathrm{~S}_{2}[\mathrm{M}-\mathrm{H}]^{+}$: 586.20, found: 585.25.

2-Cyano-3-(9-octyl-6-phenyl-9H-carbazol-3-yl)-acrylic acid (PC). Product: yellow colored solid, yield: $92 \%$. Melting point: $204{ }^{\circ} \mathrm{C}$ ${ }^{1} \mathrm{H}$ NMR, (400 MHz, DMSO- $d_{6}$ ): $\delta=8.84(\mathrm{~s}, 1 \mathrm{H}), 8.38(\mathrm{~d}, J=4.4$ $\mathrm{Hz}, 2 \mathrm{H}), 8.25$ (d, $J=8.4 \mathrm{~Hz}, 1 \mathrm{H}), 7.79-7.76(\mathrm{~m}, 2 \mathrm{H}), 7.71(\mathrm{~d}, J=8$ $\mathrm{Hz}, 3 \mathrm{H}), 7.44(\mathrm{t}, J=7.6 \mathrm{~Hz}, 2 \mathrm{H}), 7.30(\mathrm{t}, J=7.2 \mathrm{~Hz}, 1 \mathrm{H}), 4.42(\mathrm{t}, J=$ $6.8 \mathrm{~Hz}, 2 \mathrm{H}), 1.74-1.72(\mathrm{~m}, 2 \mathrm{H}), 1.20-1.11(\mathrm{~m}, 10 \mathrm{H}), 0.74(\mathrm{t}, J=6.4$ $\mathrm{Hz}, 3 \mathrm{H}) \mathrm{ppm} .{ }^{13} \mathrm{C}$ NMR, (100 MHz, DMSO- $\left.d_{6}\right): \delta=164.11,155.04$, $143.05,140.63$, 140.14, 132.71, 128.88, 127.58, 126.71, 125.97, $125.81,122.61,122.38,118.53,117.22,110.52,110.34,98.43$, 42.66, 31.10, 28.63, 28.53, 28.45, 26.31, 21.94, 13.81 ppm. MS (ESI) $m / z$ : calculated for $\mathrm{C}_{30} \mathrm{H}_{30} \mathrm{~N}_{2} \mathrm{O}_{2}[\mathrm{M}+\mathrm{H}]^{+}:$450.23, found: 449.17 .
[5-(9-Octyl-6-phenyl-9H-carbazol-3-ylmethylene)-4-oxo-2-thioxothiazolidin-3-yl]-acetic acid (PR). Product: red colored solid, yield: 86\%. Melting point: $92{ }^{\circ} \mathrm{C}^{1} \mathrm{H}$ NMR, (400 MHz, DMSO- $\left.d_{6}\right): \delta=8.59$ $(\mathrm{s}, 1 \mathrm{H}), 8.50(\mathrm{~s}, 1 \mathrm{H}), 7.98(\mathrm{~s}, 1 \mathrm{H}), 7.80-7.68(\mathrm{~m}, 5 \mathrm{H}), 7.44(\mathrm{t}, J=7.2$ $\mathrm{Hz}, 2 \mathrm{H}), 7.30(\mathrm{t}, J=7.2 \mathrm{~Hz}, 1 \mathrm{H}), 4.70(\mathrm{~s}, 2 \mathrm{H}), 4.41(\mathrm{t}, J=6.8 \mathrm{~Hz}, 2 \mathrm{H})$, $1.74(\mathrm{t}, J=6.4 \mathrm{~Hz}, 2 \mathrm{H}), 1.21-1.11(\mathrm{~m}, 10 \mathrm{H}), 0.74(\mathrm{t}, J=6.4 \mathrm{~Hz}, 3 \mathrm{H})$ ppm. ${ }^{13} \mathrm{C}$ NMR, (100 MHz, DMSO- $\left.d_{6}\right): \delta=193.65,167.78,166.90$, $142.41,141.17,140.71,136.15,132.97,129.61,129.32,128.82$, $127.27,127.19,127.15,126.23,124.75,124.16,123.72,123.04$, $119.47,117.76,111.08,110.84$, 55.32, 45.42, 43.15, 31.59, 29.12, 29.01, 28.95, 26.85, 22.43, $14.31 \mathrm{ppm}$. MS (ESI) $\mathrm{m} / \mathrm{z}$ : calculated for $\mathrm{C}_{32} \mathrm{H}_{32} \mathrm{~N}_{2} \mathrm{O}_{3} \mathrm{~S}_{2}[\mathrm{M}-\mathrm{H}]^{+}$: 556.19, found: 555.25.

2-Cyano-3-(9-octyl-9H-carbazol-3-yl)-acrylic acid (CC). Product: yellow colored solid, yield: $59 \%$. Melting point: 154 ${ }^{\circ} \mathrm{C}{ }^{1} \mathrm{H} \mathrm{NMR},\left(400 \mathrm{MHz}, \mathrm{CDCl}_{3}-d\right): \delta=8.78(\mathrm{~s}, 1 \mathrm{H}), 8.48(\mathrm{~s}, 1 \mathrm{H})$, $8.26(\mathrm{~d}, J=8.4 \mathrm{~Hz}, 1 \mathrm{H}), 8.16(\mathrm{~d}, J=7.6 \mathrm{~Hz}, 1 \mathrm{H}), 7.54(\mathrm{t}, J=8$ $\mathrm{Hz}, 1 \mathrm{H}), 7.47(\mathrm{t}, J=8.8 \mathrm{~Hz}, 2 \mathrm{H}), 7.34(\mathrm{t}, J=7.6 \mathrm{~Hz}, 1 \mathrm{H}), 4.33(\mathrm{t}$, $J=7.2 \mathrm{~Hz}, 2 \mathrm{H}), 1.89(\mathrm{p}, J=7.3 \mathrm{~Hz}, 2 \mathrm{H}), 1.41-1.21(\mathrm{~m}, 10 \mathrm{H})$, $0.86(3, J=6.4 \mathrm{~Hz}, 3 \mathrm{H}) \mathrm{ppm} .{ }^{13} \mathrm{C} \mathrm{NMR},\left(100 \mathrm{MHz}, \mathrm{CDCl}_{3}-d\right)$ : $\delta=168.99,157.42,143.68,141.11,129.54,127.04,125.86$, 123.59, 122.73, 122.32, 120.96, 120.75, 116.54, 109.53, 109.47, $96.34,43.47,31.76,29.30,29.14,28.94,27.25,22.60,14.06$ ppm. HRMS (ESI) $m / z$ : calculated for $\mathrm{C}_{24} \mathrm{H}_{26} \mathrm{~N}_{2} \mathrm{O}_{2}[\mathrm{M}-\mathrm{H}]^{+}$: 374.20 , found: 373.17 .

3-\{6-[Bis-(4-methoxy-phenyl)-amino]-9-octyl-9H-carbazol-3-yl\}2-cyano-acrylic acid (OMNC). Yield: $66.6 \%$. ${ }^{1} \mathrm{H}$ NMR $(400 \mathrm{MHz}$, $\left.\mathrm{CDCl}_{3}-d\right): \delta 8.44(\mathrm{~d}, J=6.3 \mathrm{~Hz}, 2 \mathrm{H}), 8.40(\mathrm{~d}, J=1.6 \mathrm{~Hz}, 1 \mathrm{H}), 7.79$ $(\mathrm{d}, J=1.8 \mathrm{~Hz}, 1 \mathrm{H}), 7.46(\mathrm{~d}, J=8.8 \mathrm{~Hz}, 1 \mathrm{H}), 7.37-7.29(\mathrm{~m}, 2 \mathrm{H})$, $7.10-7.00(\mathrm{~m}, 4 \mathrm{H}), 6.88-6.79(\mathrm{~m}, 4 \mathrm{H}), 4.31(\mathrm{t}, J=7.1 \mathrm{~Hz}, 2 \mathrm{H}), 3.83$ $(\mathrm{s}, 6 \mathrm{H}), 1.96-1.87(\mathrm{~m}, 2 \mathrm{H}), 1.38-1.28(\mathrm{~m}, 10 \mathrm{H}), 0.90(\mathrm{~d}, J=6.6 \mathrm{~Hz}$, $3 \mathrm{H}) .{ }^{13} \mathrm{C}$ NMR $\left(101 \mathrm{MHz}, \mathrm{CDCl}_{3}-d\right): \delta=168.09,157.32,155.06$, $143.97,142.89,142.21,136.90,128.13,127.43,124.96,124.38$, 123.62, 123.19, 122.19, 116.68, 115.81, 114.66, 110.15, 109.67, $96.23,55.55,43.63,31.85,29.72,29.52,29.48,29.36,29.27,29.06$, 28.98, 27.26, 22.67, 14.15, 14.13 .

3-\{6-[Bis-(4-hexyloxy-phenyl)-amino]-9-octyl-9H-carbazol-3-yl\}2-cyano-acrylic acid (CNC). Yield: $68.5 \% .{ }^{1} \mathrm{H}$ NMR $(400 \mathrm{MHz}$, $\left.\mathrm{CDCl}_{3}-d\right): \delta 8.42(\mathrm{~s}, 3 \mathrm{H}), 7.78(\mathrm{~s}, 1 \mathrm{H}), 7.46(\mathrm{~d}, J=9.3 \mathrm{~Hz}, 1 \mathrm{H}), 7.30$ $(\mathrm{d}, J=6.9 \mathrm{~Hz}, 2 \mathrm{H}), 7.04(\mathrm{~d}, J=8.7 \mathrm{~Hz}, 4 \mathrm{H}), 6.83(\mathrm{~d}, J=8.8 \mathrm{~Hz}$, 

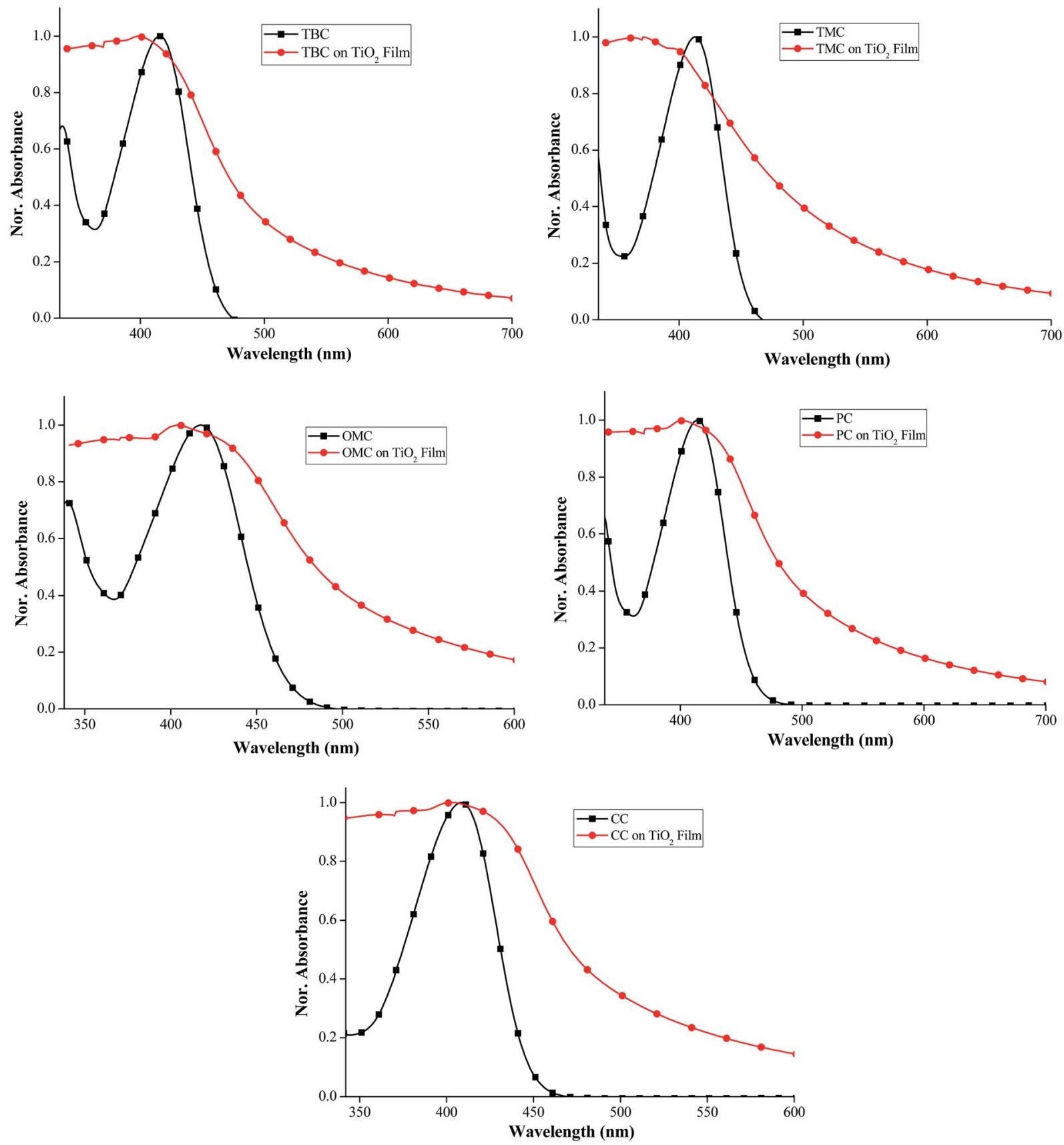

Fig. 2 Normalised absorption spectrum of TBC, TMC, OMC, PC and $\mathrm{CC}$ dyes in $\mathrm{CHCl}_{3}$ solution and on $\mathrm{TiO}_{2}$ films.

$4 \mathrm{H}), 4.31(\mathrm{t}, J=7.0 \mathrm{~Hz}, 2 \mathrm{H}), 3.96(\mathrm{t}, J=6.5 \mathrm{~Hz}, 4 \mathrm{H}), 2.39(\mathrm{~s}, 2 \mathrm{H})$, $2.04(\mathrm{~s}, 2 \mathrm{H}), 1.80(\mathrm{~s}, 2 \mathrm{H}), 1.35(\mathrm{dd}, J=13.0,6.0 \mathrm{~Hz}, 22 \mathrm{H}), 0.95-$ $0.90(\mathrm{~m}, 9 \mathrm{H}) .{ }^{13} \mathrm{C}$ NMR (101 MHz, $\left.\mathrm{CDCl}_{3}\right): \delta=179.43,168.59$, 157.31 , 154.68, 143.98, 143.02, 142.02, 136.82, 130.03, 129.73, $128.01,127.53,124.99,124.30,123.60,123.22$, 122.13, 116.59, 115.64, 115.25, 110.10, 109.67, 96.18, 77.33, 77.01, 76.69, 68.32, 43.63 , 33.93, 31.93, 31.85, 31.63, 29.70, 29.68, 29.65, 29.60, 29.51, $29.47,29.44,29.38,29.34,29.25,29.15,29.07,27.26,25.79,24.70$, 22.70, 22.66, 22.63, 14.13, 14.11, 14.06 .

6-[Bis(20,40-bis(hexyloxy)-[1,10-biphenyl]-4-yl)amino]-9-octyl9H-carbazol-3-yl\}-2-cyano-acrylic acid (HNC). Yield: $67.6 \% .{ }^{1} \mathrm{H}$ NMR (400 MHz, $\left.\mathrm{CDCl}_{3}-d\right): \delta 8.48(\mathrm{~s}, 1 \mathrm{H}), 8.45(\mathrm{~d}, J=7.2 \mathrm{~Hz}, 2 \mathrm{H})$, $8.04(\mathrm{~d}, J=1.9 \mathrm{~Hz}, 1 \mathrm{H}), 7.59-7.35(\mathrm{~m}, 8 \mathrm{H}), 7.31(\mathrm{~s}, 1 \mathrm{H}), 7.17(\mathrm{~d}, J$ $=8.7 \mathrm{~Hz}, 4 \mathrm{H}), 6.57(\mathrm{dd}, J=6.6,2.3 \mathrm{~Hz}, 4 \mathrm{H}), 4.35(\mathrm{~s}, 2 \mathrm{H}), 4.00(\mathrm{dt}$, $J=9.5,6.6 \mathrm{~Hz}, 8 \mathrm{H}), 1.87-1.70(\mathrm{~m}, 10 \mathrm{H}), 1.44-1.31(\mathrm{~m}, 34 \mathrm{H})$, 0.95-0.87 (m, 15H). ${ }^{13} \mathrm{C}$ NMR (101 $\left.\mathrm{MHz} \mathrm{CDCl}_{3}-d\right): \delta=179.48$, 168.39, 159.43, 157.24, 156.96, 146.61, 144.03, 141.68, 137.87, $132.04,130.83,130.11,129.75,127.99,127.68,126.57,123.79$, 123.24, 123.13, 122.35, 118.68, 110.33, 109.75, 105.28, 100.42, $77.36,77.24,77.04,76.72,68.40,68.12,43.70,33.95,31.95$, $31.87,31.64,31.46,29.72$, 29.62, 29.54, 29.50, 29.38, 29.34, $29.28,29.09,27.31,27.24,25.79,25.76,24.72,22.72,22.68$, $22.65,22.58,14.13,14.08,14.04,13.67$. 


\section{Results and discussion}

\subsection{Synthesis and characterization}

Schemes 1 and 2 shows the structures of carbazole based sensitizers having donor fragments with different strength. The synthetic route of CBAI is shown in scheme 3. CB is prepared from carbazole via $\mathrm{N}$-alkylation with 1-bromo octane, followed by Vilsmeier hack formylation reaction to get CBA. Iodination of CBA using $\mathrm{KI}$ and $\mathrm{KIO}_{3}$ afforded CBAI. Four different types of aryl boronic acid (4-tert-butylphenylboronic acid, (2,4,6-trimethylphenyl)boronic acid, 4-methoxyphenylboronic acid and 4-phenylboronic acid) were coupled with CBAI via Suzuki coupling reaction to get their corresponding aldehydes. A peak around 10.09-10.1 ppm in proton NMR clearly shows the presence of the aldehyde group. Knoevenagel condensation of aldehydes precursors with cyano acetic acid or rhodanine acetic acid attains the target compounds as shown in Scheme 4. CBAI undergoes Buchwald coupling with three different types of aryl amines to afford the respective aldehydes followed by Knoevenagel condensation to obtain final compounds as depicted in Scheme 5. Similarly CBA (absence of donating group) undergo Knoevenagel condensation with cyano acetic acid in the presence of ammonium acetate and acetic acid to give CC. These new molecules are characterized by ${ }^{1} \mathrm{H},{ }^{13} \mathrm{C}$ NMR, melting point measurement and mass spectroscopy techniques.

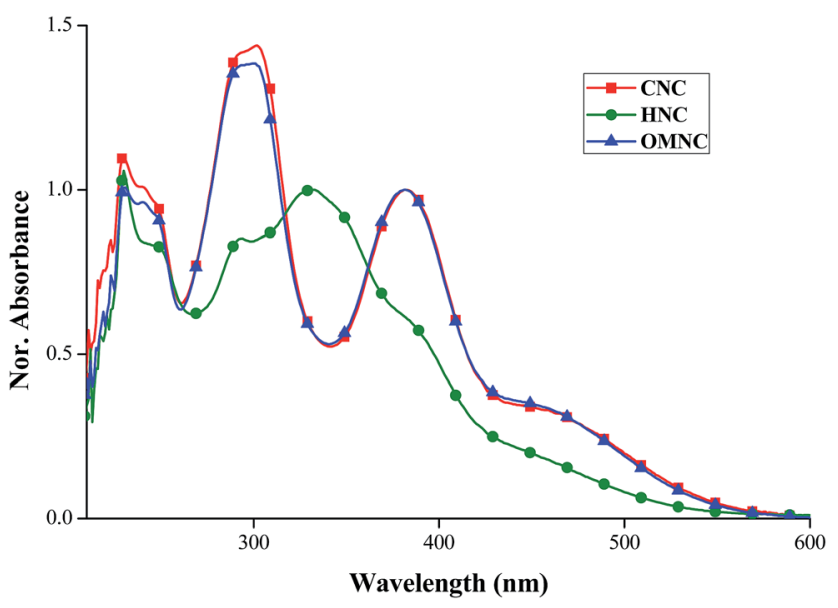

Fig. 3 Normalised absorption spectrum of amine donor based carbazole dyes in $\mathrm{CHCl}_{3}$ solutions.

\subsection{Optical properties}

Fig. 1A and B shows the absorption spectra of phenyl donor substituted sensitizers in chloroform solvent and the data are summarized in Table 1. All the sensitizers shows two types of absorption bands, one at higher energy around 250-360 nm, which arise from $\pi-\pi^{*}$ transition and another band at lower energy appears at $360-540 \mathrm{~nm}$ which can be assigned to intramolecular charge transfer transition from electron donating moiety (CTB, CTM, COMe, CPB) to electron acceptor moiety (CAA, RA). ${ }^{21}$ Due to the difference in acceptor groups (CAA or RA), the intramolecular charge transfer bands occurred in different wavelength, e.g. 408 to $418 \mathrm{~nm}$ for CAA acceptor groups and 453 to $457 \mathrm{~nm}$ for RA acceptor groups (Fig. S1 $\dagger$ ). While analyzing the effect of various substituents in the phenyl donor on carbazole unit, we didn't observe any major difference in the absorption spectrum, only slight shifts are observed. By varying the donor moiety on carbazole unit the higher energy band is red shifting in the order of CC $(408 \mathrm{~nm})<$ TMC $(413 \mathrm{~nm})$ $<$ PC $(414 \mathrm{~nm})<$ TBC $(416 \mathrm{~nm})<$ OMC $(418 \mathrm{~nm})$ for cyano acrylic acid substituted carbazole sensitizers and when rhodanine is used as acceptor, an order of TMR $(453 \mathrm{~nm})<\mathbf{P R}(454 \mathrm{~nm})<\mathbf{T B R}$ (456 nm) < OMR (457 nm) is observed. Among all compounds, methoxyphenyl incorporated sensitizer (OMC) shows the most red-shifted absorption wavelength. TBR with tert-butyl phenyl as donating moiety shows bathochromic absorption shift by 3 $\mathrm{nm}$ compared to TMC. Due to the introduction of methoxy groups in para position of phenyl ring, OMC $\left(\lambda_{\max }=418 \mathrm{~nm}, \varepsilon=\right.$ $\left.7.66 \times 10^{5} \mathrm{M}^{-1} \mathrm{~cm}^{-1}\right)$ shows three times higher molar extinction coefficient than PC $\left(\lambda_{\max }=414 \mathrm{~nm}, \varepsilon=4.36 \times 10^{5} \mathrm{M}^{-1}\right.$ $\left.\mathrm{cm}^{-1}\right)$ The molar extinction coefficient of TBC $\left(\lambda_{\max }=416 \mathrm{~nm}, \varepsilon\right.$ $\left.=2.66 \times 10^{5} \mathrm{M}^{-1} \mathrm{~cm}^{-1}\right)$ are slightly higher than TMC $\left(\lambda_{\max }=\right.$ $\left.413 \mathrm{~nm}, \varepsilon=1.97 \times 10^{5} \mathrm{M}^{-1} \mathrm{~cm}^{-1}\right)$ and CC $\left(\lambda_{\max }=408 \mathrm{~nm}, \varepsilon=\right.$ $\left.1.70 \times 10^{5} \mathrm{M}^{-1} \mathrm{~cm}^{-1}\right)$. RA derivatives shows molar extinction coefficient in the range of $\left(1.66\right.$ to $\left.3.23 \times 10^{5} \mathrm{M}^{-1} \mathrm{~cm}^{-1}\right)$. It is worth noting that inspite of simple structures of these sensitizers, their molar extinction coefficient are significantly higher than those of ruthenium sensitizers. ${ }^{22}$

The absorption behavior of the sensitizers attached to the $\mathrm{TiO}_{2}$ surface is depicted in Fig. 2. Those absorption spectra are broadened and blue shifted as compared in solution state due to the strong interaction between dyes and semiconductor surface. Hypsochromic shift of $5 \mathrm{~nm}$ to $42 \mathrm{~nm}$ for cyano acrylic acid based compounds (OMC, PC, TBC, CC and TMC) occurred due to the formation of $\mathrm{H}$ type aggregation or deprotonation of the dyes on the surface of the $\mathrm{TiO}_{2}$ films. ${ }^{17,23}$ Rhodanine acetic

Table 2 Absorption, emission and electrochemical properties of amine donor based carbazole dyes

\begin{tabular}{llllll}
\hline & $\lambda_{\max }{ }^{a}(\mathrm{~nm}) /\left[\varepsilon\left(\mathrm{M}^{-1} \mathrm{~cm}^{-1}\right)\right]$ & $\lambda_{\mathrm{emi}}{ }^{a}(\mathrm{~nm})$ & $E_{\mathrm{ox}}{ }^{b}(\mathrm{~V}) v s . \mathrm{NHE}$ & $E_{0-0}{ }^{c}(\mathrm{eV}) v s . \mathrm{NHE}$ & $E_{\text {ox }}-E_{0-0}(\mathrm{~V})$ \\
\hline OMNC & $382(4560) 455(1560)$ & 493 & 0.73 & 2.81 \\
CNC & $382(17900) 459(5940)$ & 618 & 1.18 & 2.92 & -2.08 \\
HNC & $331(67200) 387(39300)$ & 513 & 0.83 & 2.75
\end{tabular}

${ }^{a}$ Absorption and emission spectrum of amine donor based carbazole dyes were measured in $\mathrm{CHCl}_{3}$ solutions. ${ }^{b}$ Cyclic voltammograms of the first oxidation potential of the dyes were measured in dichloromethane solution containing $0.1 \mathrm{M} \mathrm{TBAPF}_{6}$ used as an supporting electrolyte (working electrode: Pt, counter electrode: Pt wire, reference electrode: $\mathrm{Ag} / \mathrm{AgCl}$ calibrated with ferrocene/ferrocenium $\left(\mathrm{Fc} / \mathrm{Fc}^{+}\right)$as an internal reference and converted to NHE by adding $630 \mathrm{mV}$ ). ${ }^{c}$ The $E_{0-0}$ transition values was estimated from the onset of absorption and emission spectra. 

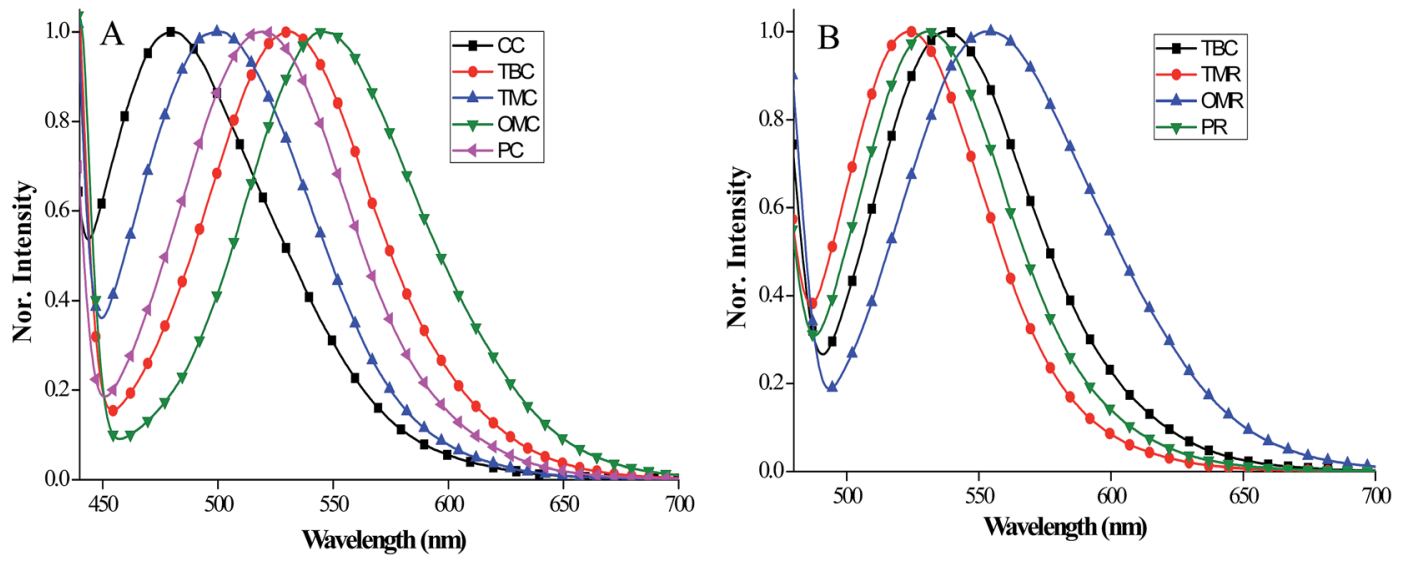

Fig. 4 Normalised fluorescence spectrum of phenyl donor based carbazole dyes in $\mathrm{CHCl}_{3}$ solutions.

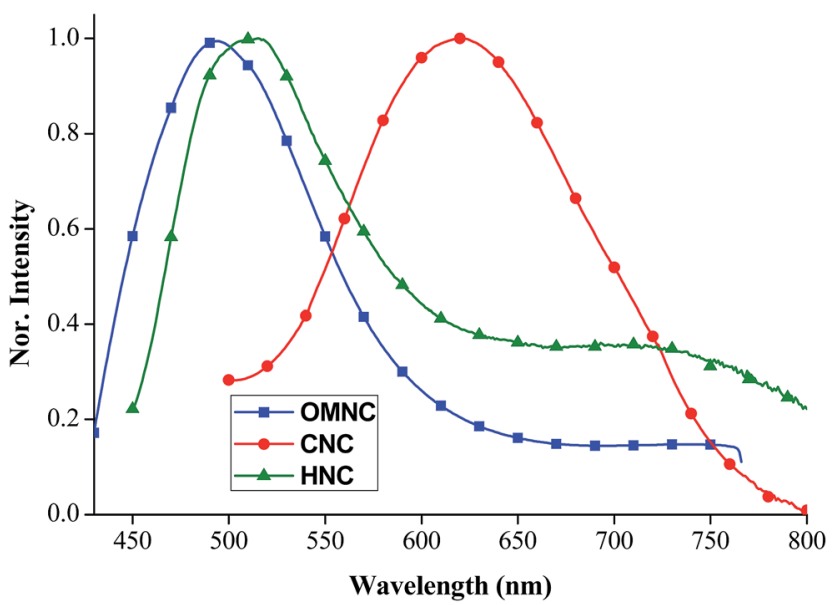

Fig. 5 Normalised fluorescence spectrum of amine donor based carbazole dyes in $\mathrm{CHCl}_{3}$ solutions.

acid based dyes (TBR, TMR, OMR and PR) shows more prominent blue shift by $\sim 86 \mathrm{~nm}$ owing to the strong deprotonation of carboxylic acid onto the $\mathrm{TiO}_{2}$ surface (Fig. S2 $\dagger$ ). While comparing these two acceptor moieties, rhodanine acetic acid substituted compounds absorb at longer wavelength than that of cyano acrylic acid based compounds both in chloroform solution and $\mathrm{TiO}_{2}$ film.

To further increase the donor strength, we choose phenyl amine based donor groups. Aryl amine donors with different alkyl chain substitution were synthesized to afford OMNC, CNC and HNC. Fig. 3 shows the absorption spectra of OMNC, CNC and $\mathbf{H N C}$ and the data is displayed in Table 2. Firstly, OMNC with para-substituted methoxy groups on diphenylamine shows the maximum absorption at 382 and $455 \mathrm{~nm}$. By increasing the alkyl chain from methoxy to hexyloxy, a slightly red shifted absorption is observed at 382 and $459 \mathrm{~nm}$. However, by introducing one more phenyl group with two hexyloxy groups attached in ortho and para positions leads to blue shifted absorption at $331 \mathrm{~nm}$, which indicates the decreased donating strength of amine donor. Replacing phenyl donors with stronger amine donor results in red shifted onset absorption values which appear around $570 \mathrm{~nm}$. Among sensitizers with phenyl donors the onset values appeared around $490 \mathrm{~nm}$ for CAA anchoring group and $530 \mathrm{~nm}$ for RA anchoring unit. The maximum absorption coefficient of the amine donor based sensitizers are in the order of HNC $\left(\varepsilon=3.93 \times 10^{4} \mathrm{M}^{-1} \mathrm{~cm}^{-1}\right.$ at $387 \mathrm{~nm})>\mathbf{C N C}\left(\varepsilon=5.94 \times 10^{3} \mathrm{M}^{-1} \mathrm{~cm}^{-1}\right.$ at $\left.459 \mathrm{~nm}\right)>$ OMNC $(\varepsilon$ $=1.56 \times 10^{3} \mathrm{M}^{-1} \mathrm{~cm}^{-1}$ at $\left.455 \mathrm{~nm}\right)$. Increasing bulkiness via alkylation in the donor fragment prevents the dye aggregation which increases their molar absorption coefficient. The emission spectra of all carbazole sensitizers are recorded in chloroform and displayed in Fig. 4A and B. The emission data are collected in Table 1 and the values of different donor compounds are appeared in the same order of absorption maximum. Fluorescence spectra of amine donor dyes are displayed in Fig. 5 and the data is given in Table 2. When we look over the absorption spectra, a similar maximum absorption peak is observed for OMNC and CNC. However, the maximum emission peak of CNC is observed at $618 \mathrm{~nm}$ which is $125 \mathrm{~nm}$ higher than OMNC (493 nm). This reveals that the presence of alkyl chains in the donor fragment have significant impact in changing the excited state of the molecule.

\subsection{Electrochemical properties}

The first oxidation potential of the carbazole based sensitizers corresponds to the ground state potential level (i.e. HOMO) of the dye and is important for their photovoltaic applications. Cyclic voltammetry measurements were carried out to analyze the HOMO level of the dyes using tetrabutyl ammonium hexafluoro phosphate $\left(\mathrm{TBAPF}_{6}\right)$ as the supporting electrolyte. Ferrocene/ferrocenium $\left(\mathrm{Fc} / \mathrm{Fc}^{+}\right)$is used as an internal standard. The electrochemical data of all carbazole sensitizers are presented in Fig. 6 and summarized in Table 1. Single oxidation peaks are observed for $\mathbf{C C}, \mathbf{P C}, \mathbf{P R}$ and TMR and two oxidation peaks are observed for OMC, OMR, TBR and TMC and three oxidation peaks is observed for TBC. The first oxidation peaks of all dyes and second oxidation peak of OMC, OMR and TMC are reversible in nature, which suggest the oxidized states of the dyes are quite stable. ${ }^{24}$ PC, TBR and TMR display quasireversible second oxidation peaks. The first oxidation peaks of 

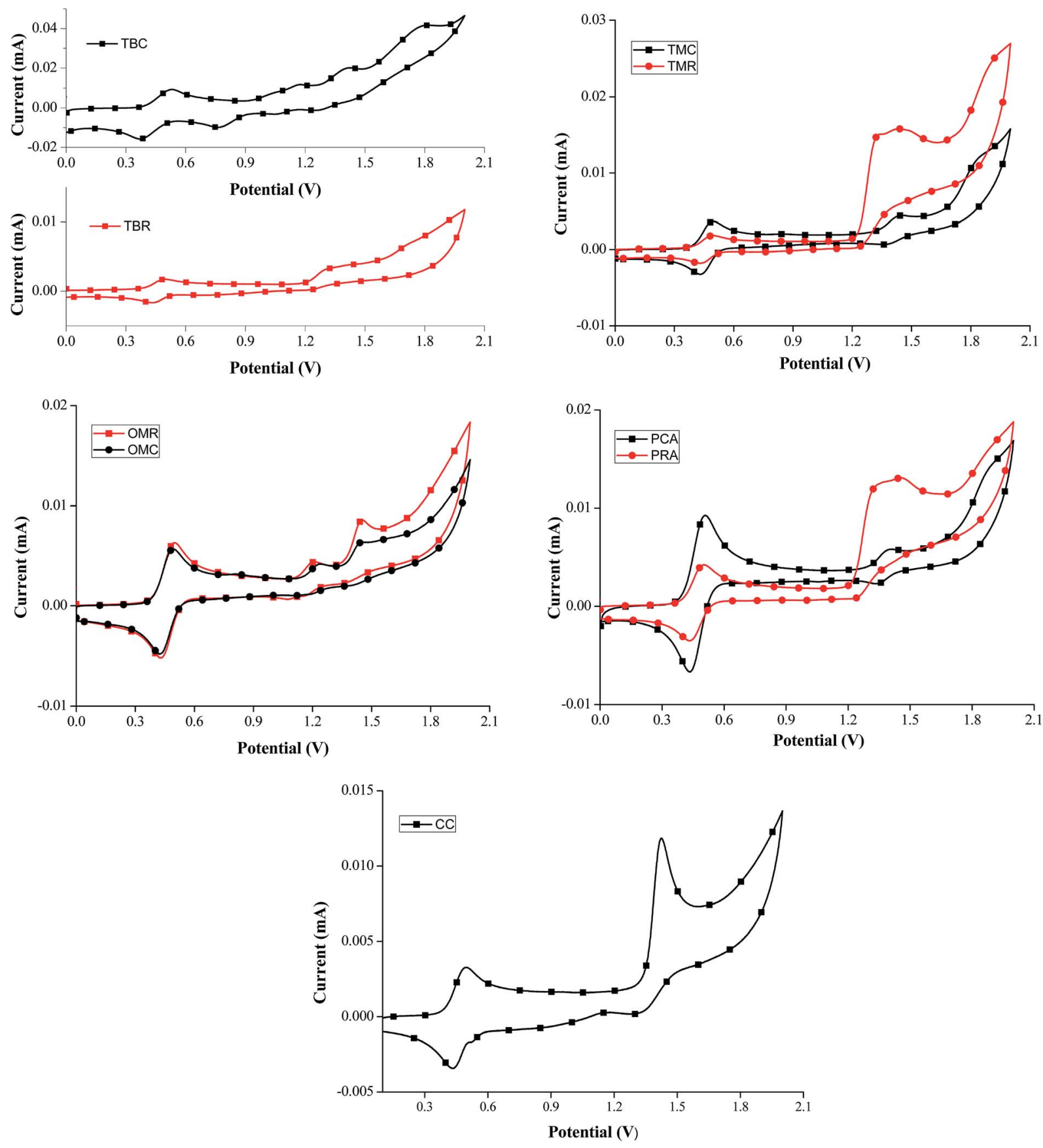

Fig. 6 Cyclic voltammograms of the phenyl donor based carbazole dyes in acetonitrile solutions.

all dyes occurs in the range of $1.15-1.58 \mathrm{~V}$, which corresponds to the removal of electron from the donor moiety and second oxidation peaks occur at higher potentials, which can be attributed to the removal of electron from conjugated backbone of the dyes. ${ }^{25}$ The oxidation potential of the sensitizers with amine donor in dichloromethane solutions are depicted in Fig. 7 and Table 2. The first oxidation potentials of OMNC, CNC and $\mathrm{HNC}$ are $0.73 \mathrm{~V}, 1.18 \mathrm{~V}$ and $0.83 \mathrm{~V}$, respectively. All the oxidation peaks are reversible in nature, showing that the oxidized states of these dyes are quite stable. The HOMO of the amine donor dyes occurs at lower potential than the phenyl donor dyes which is due to the strong donating ability of amine donor which results in easy removal of electrons and oxidise at lower potential. The HOMO level of the dyes are more positive than $\mathrm{I}^{-} / \mathrm{I}_{3}{ }^{-}$electrolyte $(0.4 \mathrm{~V} v s$. NHE), which reveals the efficient dye regeneration from electrolytes.

The excited state oxidation potential (i.e. LUMO) can be calculated by subtracting zero-zero excitation energy $\left(E_{0-0}\right)$ from HOMO level of the dyes. The $E_{0-0}$ energy values can be obtained from the point of intersection of the experimental absorption and emission spectra of sensitizers and the data is displayed in Table 1. The obtained LUMO values are sufficient for electron 

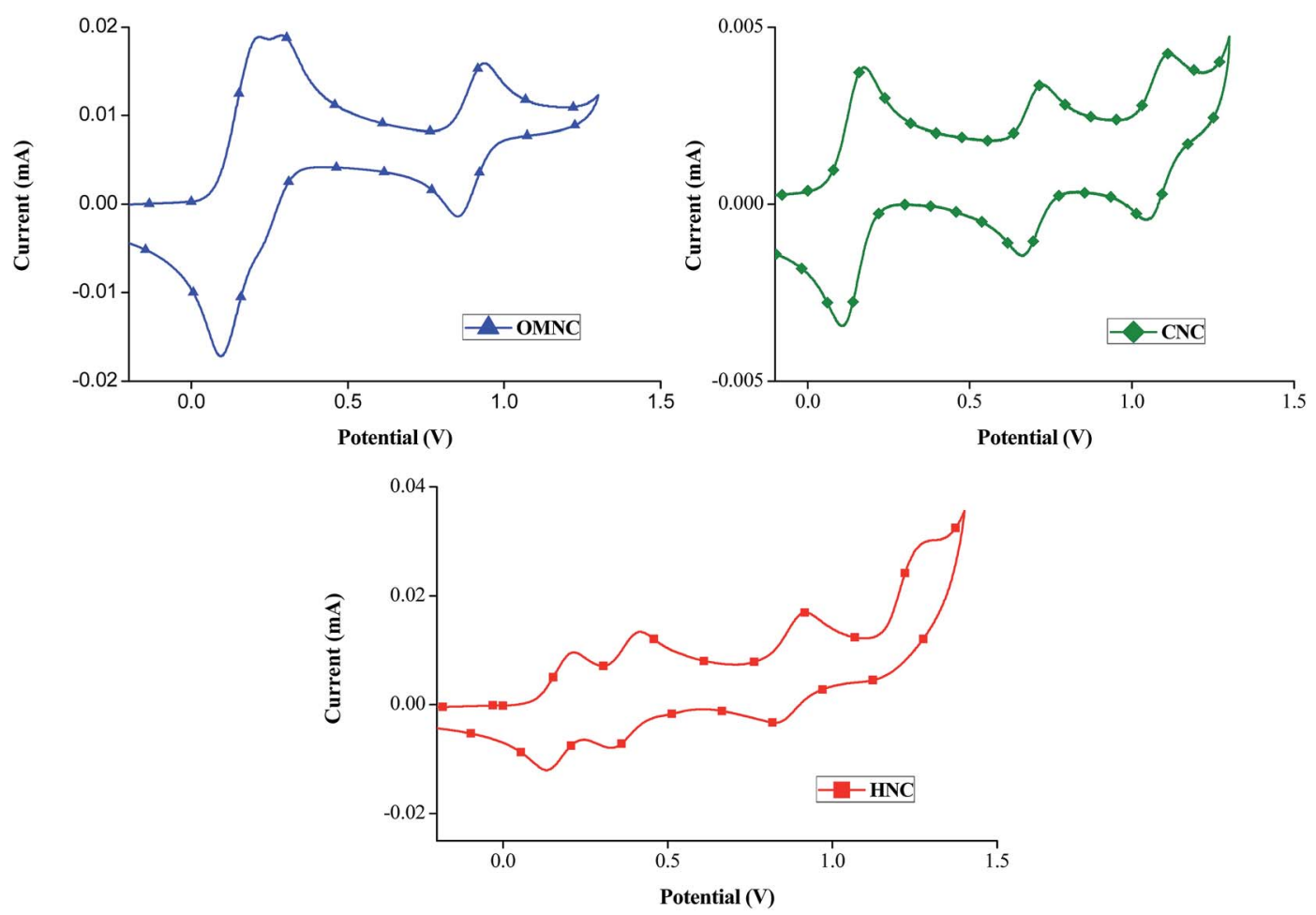

Fig. 7 Cyclic voltammograms of the phenyl donor based carbazole dyes in dichloromethane solutions.

injection into conduction band potential of $\mathrm{TiO}_{2}(-0.5 \mathrm{~V} v$. NHE). The electrochemical data supports the thermodynamic driving force for efficient dye regeneration and electron injection in DSSC (Fig. 8).

The geometries of the dyes are optimized by density functional theory (DFT) calculations at the B3LYP.6-31G(d) ${ }^{21}$ level to analyze further the distribution of HOMO and lowest unoccupied molecular orbital (LUMO) energy levels of the sensitizers. Fig. S3A-F and Table $\mathrm{S} 1 \uparrow$ display the electron distributions of the HOMO and LUMO of the dyes. HOMOs are mainly delocalized in the phenyl and carbazole groups in the phenyl based donor fragment, while it resides mainly on the amino substituents in the amine based donor group. LUMOs show localized electron distributions on the acceptor parts cyanoacrylic acid and rhodanine acetic acid. The observed spatially separated frontier orbitals strongly promote intramolecular charge separation and hence favor efficient electron injection from the excited state of the dye into the semiconducting oxide.

\subsection{Photovoltaic properties}

5.4.1. IV characterization. The impact of different donors on solar energy conversion is analyzed by employing the sensitizers in DSSC with iodine iodide redox electrolyte. The photocurrent density-photovoltage $(J-V)$ curves of sensitizers with phenyl and amine based donors measured under simulated AM 1.5 solar irradiation $\left(100 \mathrm{~mW} \mathrm{~cm}^{-2}\right)$ are depicted in Fig. 9A and B. The parameters such as short circuit current $\left(J_{\mathrm{SC}}\right)$, open circuit voltage $\left(V_{\mathrm{OC}}\right)$, fill factor (ff) and photovoltaic conversion efficiency $(\eta)$ data are collected in Tables $3 \& 4$.
Among the sensitizers with phenyl based donors, OMC with methoxy substituted phenyl and maximum absorption wavelength shows higher $J_{\mathrm{SC}}$ of $4.96 \mathrm{~mA} \mathrm{~cm}^{-2}$ than that $(4.15 \mathrm{~mA}$ $\mathrm{cm}^{-2}$ ) of tert-butyl phenyl substituted carbazole (TBC). For other types of substitution on the phenyl group, the $J_{\mathrm{SC}}$ only turn out to be around 2 to $2.6 \mathrm{~mA} \mathrm{~cm} \mathrm{~cm}^{-2}$. Among phenyl based donors, OMC and TBC have higher $V_{\text {OC }}$ of $730 \mathrm{mV}$. The $V_{\text {OC }}$ of OMC and TBC are higher, because the presence of methoxy and tert-butyl groups which suppresses the recombination of injected electrons by the electrolyte. Overall OMC shows higher efficiency of $2.69 \%$ and $2.17 \%$ for TBC while other shows in the range of $1 \%$. These results show that varying the substituents in the phenyl group has significant impact on the overall conversion efficiencies. The presence of trimethylphenyl (TMC) and phenyl (PC) donors shows similar efficiency $\left(J_{\mathrm{SC}}=2.06 \mathrm{~mA} \mathrm{~cm}^{-2}, V_{\mathrm{OC}}=\right.$ $0.634 \mathrm{~V}, \mathrm{ff}=0.750, \eta=0.98 \%)$ for TMC and $\left(J_{\mathrm{SC}}=2.24 \mathrm{~mA}\right.$ $\mathrm{cm}^{-2}, V_{\mathrm{OC}}=0.574 \mathrm{~V}, \mathrm{ff}=0.765, \eta=0.98 \%$ ) for PC. Interestingly, the $J_{\mathrm{SC}}$ of $\mathbf{C C}$ (no donor group) is $2.63 \mathrm{~mA} \mathrm{~cm}^{-2}$ with the efficiency of $1.11 \%$ which is higher than the substituted carbazole TMC and PC. Further comparison of the sensitizers with cyano acrylic acid and rhodanine acetic acid as acceptors, the later shows low short circuit current density. For instance, $4.96 \mathrm{~mA}$ $\mathrm{cm}^{-2}$ OMC is reduced to $2.50 \mathrm{~mA} \mathrm{~cm}^{-2}$ for OMR. This lower $J_{\mathrm{SC}}$ of RA acceptor is due to its inefficient electron injection to the $\mathrm{TiO}_{2}$. Due to the lower $J_{\mathrm{SC}}$, all the RA substituted compounds shows lower efficiency. Despite this low efficiency, RA substituted compounds shows higher fill factor among all the compounds. As expected increasing the strength of the donor from phenyl to amine leads to higher short circuit current density and power conversion efficiency. For example 


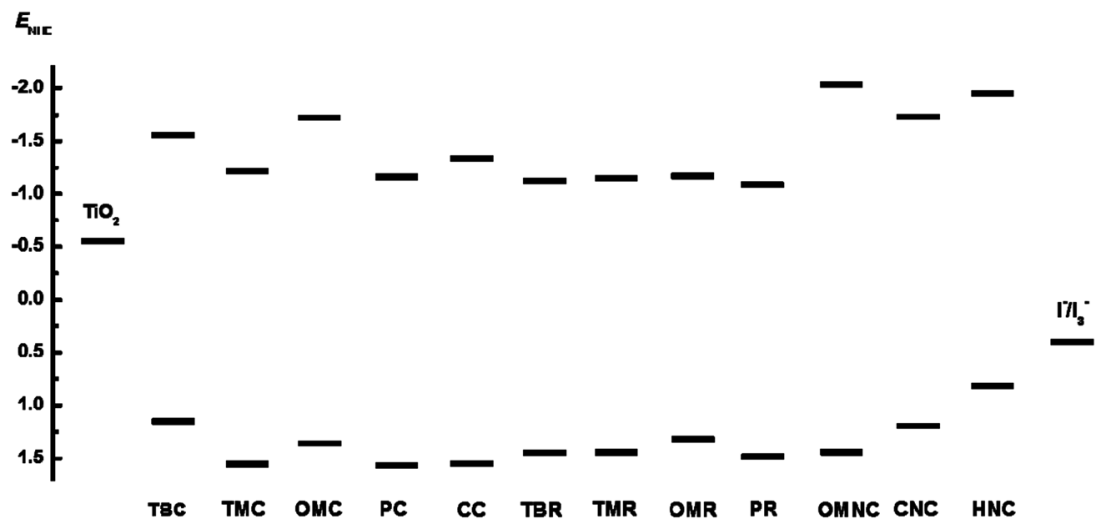

Fig. 8 Energy band gap diagrams for all the dyes in comparison to $\mathrm{TiO}_{2}$ and $\mathrm{I}^{-} / \mathrm{I}_{3}^{-}$.

sensitizers with dianisole amine as donor shows $\sim 2 \mathrm{~mA}$ higher $J_{\text {SC }}$ than OMC with only methoxy substitution. Among the different substituents in the amine donor, the hexyloxy and methoxy substituted compounds shows higher short circuit current and higher efficiency of $3.3 \%$ and $3.25 \%$ respectively. The bulky amine donor leads to lesser $J_{\mathrm{SC}}$ than the other which may be due to its lower absorbance. However changes in the substituents of the amine donor have greater impact on the open circuit potential. $V_{\mathrm{OC}}$ is obtained in the order of HNC (752 $\mathrm{mV})>$ CNC $(735 \mathrm{mV})>$ OMNC $(690 \mathrm{mV})$. Changing the methoxy substituent to hexyloxy, an increase in $V_{\mathrm{OC}}$ of $45 \mathrm{mV}$ is achieved and at the same time a higher efficiency of $3.33 \%$. Introducing more alkoxy and phenyl group in the amine donor (HNC) resulted in highest $V_{\mathrm{OC}}$ value among all carbazole dyes. This is due to the presence of more alkoxy chains in HNC helps in restricting the electrolyte to reach the $\mathrm{TiO}_{2}$ surface and thus preventing the recombination. Despite the low $V_{\mathrm{OC}}$ of OMNC than CNC, the higher fill factor of OMNC results in similar efficiency of $3.25 \%$. Although HNC shows higher fill factor than other amine donors, its lower $J_{\mathrm{SC}}$ resulted in overall conversion efficiency of $3.01 \%$.

In Fig. 9b, the dark current curves of OMNC, CNC and HNC are displayed. The dark current values of dyes are in the order of HNC $<$ OMNC $\sim$ CNC indicating that, on increase the $V_{\text {OC }}$ are beneficial to suppress the recombination of injected electrons at the interface or in the electrolyte.

5.4.2. Transient measurements. Transient photocurrent and photovoltage measurements were carried out to get further insights into the difference in the open circuit potential of the dyes OMNC, CNC and HNC which gives vital information about the effect of the alkyl chain substituents on the donor fragment. Variation in open circuit potential can possibly originate from three factors: (a) the shift in the conduction band of the $\mathrm{TiO}_{2}$ semiconductor which alters the density of occupied states (dos), (b) recombination of the injected electrons in the semiconductor to the redox mediator, and (c) the redox potential of the electrolyte species. Since the same redox mediator is used in all these dyes, we neglect this factor (c) from the discussion., ${ }^{2,26,27}$ Measurement of capacitance as a function of voltage will provide information about the conduction band of the $\mathrm{TiO}_{2}$ and the plot is depicted Fig. 10a. The results from this measurement reveal that the capacitance of lengthy alkyl chain substituted CNC and HNC is slightly shifted to higher $V_{\mathrm{OC}}$ than the methyl substituted OMNC. However the observed difference in the conduction band of $\mathrm{TiO}_{2}$ is small and suggests that this factor has minimal role in determining the open circuit potential of these devices. Hence, the recombination factor should be responsible for the observed difference in the $V_{\mathrm{OC}}$. Electron
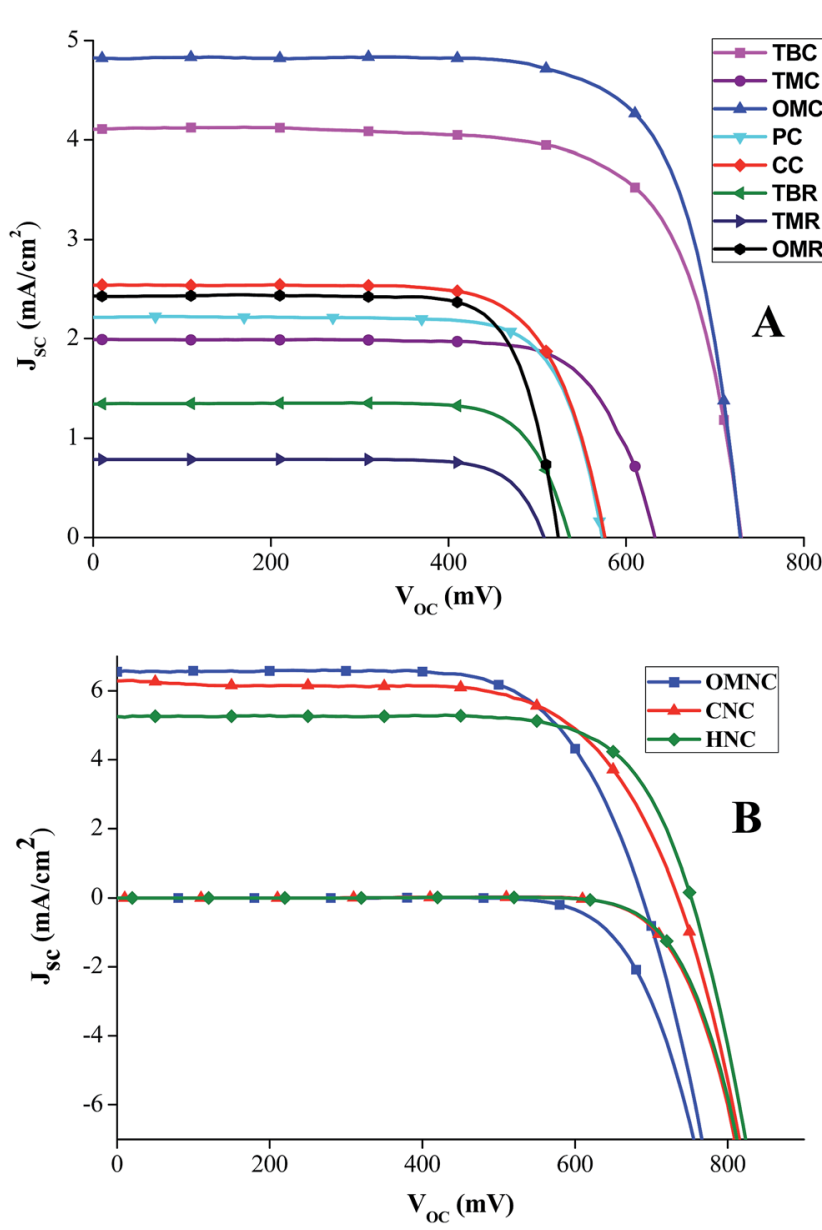

Fig. $9 J-V$ curves of DSSCs (A) phenyl donor and (B) amine donor based on carbazole sensitizers. 
Table $3 \mathrm{~J}-V$ curves of DSSC sensitized with phenyl donor based carbazole dyes in iodine electrolyte ${ }^{a}$

\begin{tabular}{lllll}
\hline & $J_{\text {SC }}\left(\mathrm{mA} \mathrm{cm}^{-2}\right)$ & $V_{\text {OC }}(\mathrm{V})$ & ff & $\eta(\%)$ \\
\hline TBC & 4.15 & 0.730 & 0.718 & 2.17 \\
TMC & 2.06 & 0.634 & 0.750 & 0.98 \\
OMC & 4.96 & 0.730 & 0.743 & 2.69 \\
PC & 2.24 & 0.574 & 0.765 & 0.98 \\
CC & 2.63 & 0.577 & 0.731 & 1.11 \\
TBR & 1.40 & 0.537 & 0.775 & 0.58 \\
TMR & 0.813 & 0.510 & 0.779 & 0.32 \\
OMR & 2.50 & 0.525 & 0.775 & 1.02
\end{tabular}

${ }^{a} \mathrm{TiO}_{2}$ film has a $8 \mu \mathrm{m}$ scattering layer and a $5 \mu \mathrm{m}$ transparent layer. Electrolyte composition: 1.0 M DMII (1,3-dimethylimidazolium iodide), $0.03 \mathrm{M}$ iodine, $0.025 \mathrm{M}$ NaI, $0.5 \mathrm{M}$ TBP, $0.1 \mathrm{M}$ guanidinium thiocyanide and acetonitrile solvent.

recombination occurring at the $\mathrm{TiO}_{2}$ electrolyte interface can be revealed by the measurement of lifetime as a function of $V_{\mathrm{OC}}$ and the plot is displayed in Fig. 10b. At a given $V_{\mathrm{OC}}$, the lifetime of alkyl chain substituted HNC and CNC is higher than the OMNC implying the slower recombination rate of the former dyes. This observation further supports the presence of bulkiness in the donor group of $\mathbf{H N C}$ prevents the electrolyte to reach the $\mathrm{TiO}_{2}$ surface that assist in reducing the recombination and achieving higher $V_{\mathrm{OC}}$. Lower lifetime of OMNC indicates its higher recombination rate that results in lower $V_{\mathrm{OC}}$. The observed trend is consistent with the observed $V_{\mathrm{OC}}$ of the devices. Transient measurements reveal that recombination is

Table 4 Photovoltaic parameters of DSSCs of amine donor based carbazole sensitizers

\begin{tabular}{lllll}
\hline & $J_{\mathrm{SC}}\left(\mathrm{mA} \mathrm{cm}^{-2}\right)$ & $V_{\text {OC }}(\mathrm{V})$ & ff & $\eta(\%)$ \\
\hline OMNC & 6.84 & 0.690 & 0.689 & 3.25 \\
CNC & 6.84 & 0.735 & 0.663 & 3.33 \\
HNC & 5.43 & 0.752 & 0.737 & 3.01
\end{tabular}

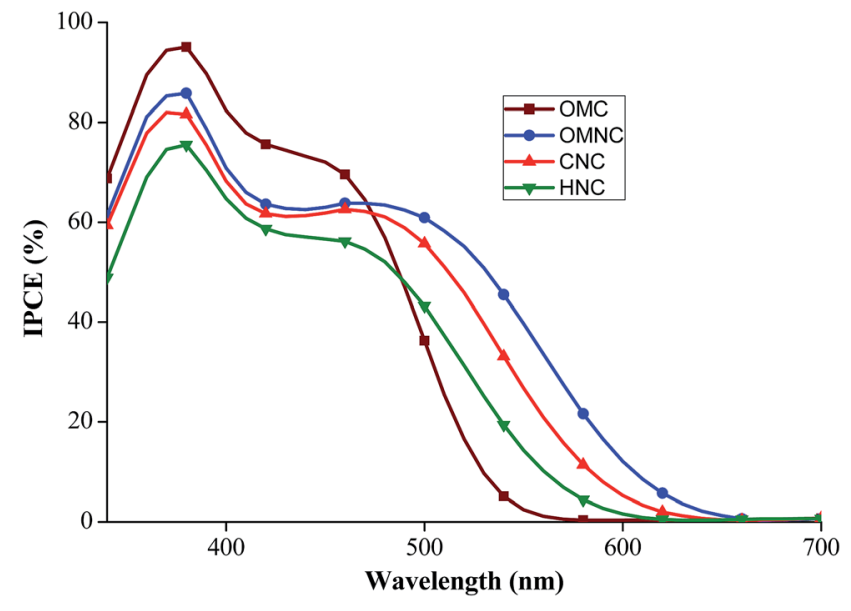

Fig. 11 IPCE spectra of DSSCs based on OMC, OMNC, CNC and HNC.

critical factor in determining the open circuit potential which found to be affected by the bulkiness in the donor fragment.

5.4.3. IPCE measurements. Based on the performance of $J-$ $V$ curves, the IPCE curves are recorded for higher $J_{\mathrm{SC}}$ dyes such as OMC, OMNC, CNC and HNC and are displayed in Fig. 11. Similar to the absorption spectra of dyes, the IPCE response shows two bands in the broad range of 340-660 $\mathrm{nm}$. The first band of IPCE based on all dyes exceeds $70 \%(350-400 \mathrm{~nm})$ and the next band exceeds $50 \%(400-520 \mathrm{~nm})$. The IPCE values exceeds $50 \%$ in the range of $340-485 \mathrm{~nm}$ for OMC, which reaches it maximum of $95 \%$ at $378 \mathrm{~nm}$ and of $72 \%$ at $450 \mathrm{~nm}$. The maximum IPCE of OMNC, CNC and HNC at $460 \mathrm{~nm}$ are $64 \%$, $62 \%$ and $56 \%$, respectively. The onset of the IPCE spectra of OMC appears at $567 \mathrm{~nm}$, OMNC at $660 \mathrm{~nm}, \mathrm{CNC}$ at $646 \mathrm{~nm}$ and $\mathrm{HNC}$ at $622 \mathrm{~nm}$. The change in IPCE behaviour of the dyes is in agreement with the absorbance behaviour. The IPCE value of OMNC are little higher than the CNC, revealing that methoxy substituted amine donor are strongly converting incident photon to current than the hexyloxy donor groups. Although, OMC (without amine donor) shows higher IPCE than OMNC (with amine donor) the former didn't cover the full spectral
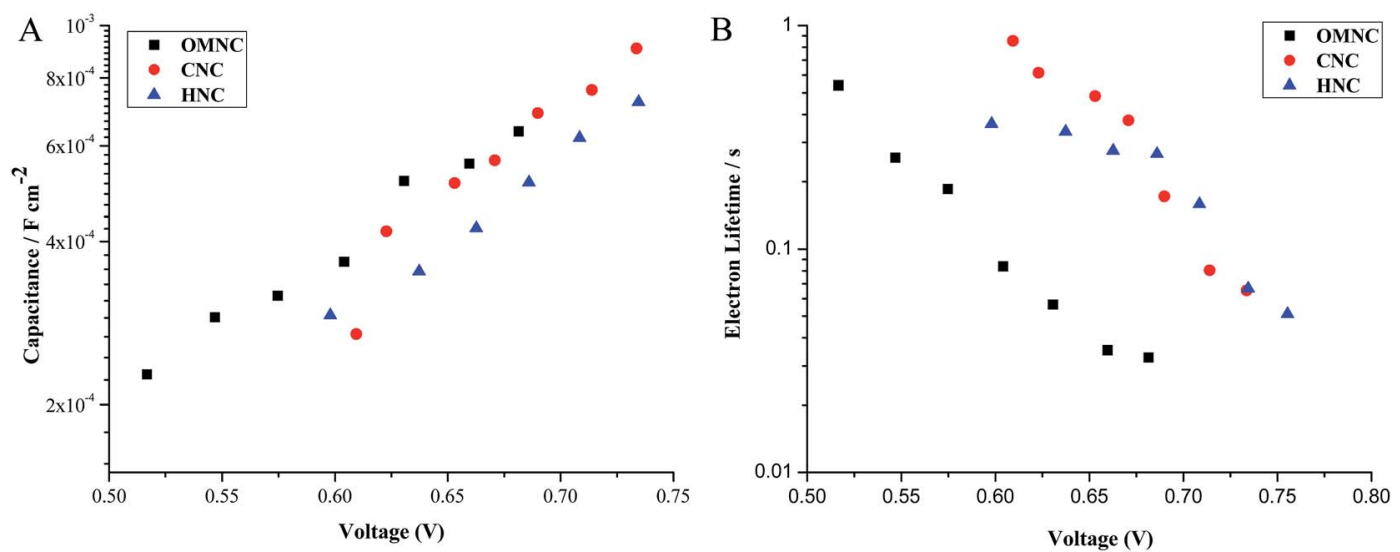

Fig. 10 (A) Chemical capacitance (B) electron lifetime as function of $V_{O C}$ obtained through transient photocurrent and photovoltage measurements of devices made with OMNC, CNC and HNC. 
region. This may be the reason for the lower performance in efficiency of OMC. The overall results suggest that the change in the donor groups have significant impact in the IPCE of the D$\pi-$ A dyes.

\section{Conclusions}

In conclusion, to understand the influence of donor groups on the optoelectronic properties, a series of carbazole based sensitizers with phenyl and amine based donor groups were successfully synthesized and characterized by ${ }^{1} \mathrm{H}$ and ${ }^{13} \mathrm{C}$ NMR, and mass spectrometric measurements. For phenyl based carbazole sensitizers, the maximum absorption appeared around $418 \mathrm{~nm}$ for methoxy substituted phenyl based donor (OMC) with CAA acceptor and $457 \mathrm{~nm}$ for RA as acceptor (OMR). In the presence of amine donor, CNC shows maximum absorption at $459 \mathrm{~nm}$ which reveals the more donating strength of amine donor substituted carbazole compounds than the phenyl donor based sensitizers. Due to $\mathrm{H}$ type aggregation of phenyl based carbazole dyes on the surface of $\mathrm{TiO}_{2}$, a hypsochromic shift is observed in the absorption spectrum of solid state films. From electrochemical data, the observed HOMO and LUMO values supports the feasible thermodynamic driving force for efficient dye regeneration and electron injection in DSSC. The DSSC made with these carbazole sensitizers displayed overall conversion efficiency in the range of $0.32-3.33 \%$. OMC shows overall conversion efficiency of $2.69 \%\left(J_{\mathrm{SC}}=4.96\right.$ $\mathrm{mA} \mathrm{cm}{ }^{-2}, V_{\mathrm{OC}}=0.730 \mathrm{mV}, \mathrm{ff}=0.743$ ) for phenyl based donor groups and CNC shows $3.33 \%\left(J_{\mathrm{SC}}=6.84 \mathrm{~mA} \mathrm{~cm}^{-2}, V_{\mathrm{OC}}=735\right.$ $\mathrm{mV}, \mathrm{ff}=0.663$ ) for amine based donor groups. RA acceptor based sensitizers shows overall poor performance due to inefficient electron injection of RA acceptor groups to $\mathrm{TiO}_{2}$. The maximum IPCE of OMNC, CNC and HNC at $460 \mathrm{~nm}$ are $64 \%$, $62 \%$ and $56 \%$, respectively. Despite the high IPCE $(72 \%$ at 450 $\mathrm{nm}$ ) of OMC than amine donor compounds, its lower absorbance region than amine based donor compounds results in lower efficiency. The open circuit potential is found to be depending on the bulkiness in the amine based donors which was probed through transient photovoltage measurements. The observed results provides vital information for future construction of donor fragments with more $\pi$-conjugated molecules to achieve wide range of absorption in the visible region. Overall, the results emphasize the varying strength and size of the donor fragment in the $\mathrm{D}-\pi-\mathrm{A}$ sensitizers have significant impact in determining the optoelectronic and photovoltaic properties.

\section{Acknowledgements}

S. J. (Ref. No. 039680/E15/2011 Dt: 17.02.2011) thanks UGC-BSR (RFSMS) for the Fellowship. G. P. thanks the Swiss government scholarship (Ref No. 2012.0795/India/OP). R. R. thanks DST-NM (Ref. No. SR/NM/NS-26/2013(G), DT: 21.10.2014) and UGC (MRP-MAJOR-CHEM-2013-35169) for the Project and UGCEmeritus fellowship (UGC-EF-7855, 2016-2017). P.G. thank acknowledge funding from the European Union Seventh Framework Programme [FP7/2007-2013] under grant agreement no. 604032 of the MESO project. The present study was performed within the Project "Establishment of the Laboratory Photoactive Nanocomposite Materials” No. 14.Z50.31.0016 supported by a Mega-grant of the Government of the Russian Federation. Authors also thank UGC/DST-FIST for NMR facilities in the School of Chemistry, Bharathidasan University.

\section{References}

1 B. O'Regan and M. Grätzel, Nature, 1991, 353, 737-740.

2 A. Hagfeldt, G. Boschloo, L. Sun, L. Kloo and H. Pettersson, Chem. Rev., 2010, 110, 6595-6663.

3 S. Mathew, A. Yella, P. Gao, R. Humphry-Baker, F. E. CurchodBasile, N. Ashari-Astani, I. Tavernelli, U. Rothlisberger, Md. K. Nazeeruddin and M. Grätzel, Nat. Chem., 2014, 6, 242-247.

4 (a) Z. S. Wang, Y. Cui, K. Hara, Y. Dan-oh, C. Kasada and A. Shinpo, Adv. Mater., 2007, 19, 1138-1141; (b) K. Hara, M. Kurashige, Y. Danoh, C. Kasada, A. Shinpo, S. Suga, K. Sayama and H. Arakawa, New J. Chem., 2003, 27, 783-785.

5 B. Liu, W. Zhu, Q. Zhang, W. Wu, M. Xu, Z. Ning, Y. Xie and H. Tian, Chem. Commun., 2009, 13, 1766-1768.

6 C. Zafer, M. Kus, G. Turkmen, H. Dincalp, S. Demic, B. Kuban, Y. Teoman and S. Icli, Sol. Energy Mater. Sol. Cells, 2007, 91, 427-431.

7 X. Ma, J. Hua, W. Wu, Y. Jin, F. Meng, W. Zhan and H. Tian, A high-efficiency cyanine dye for dye-sensitized solar cells, Tetrahedron, 2008, 64, 345-350.

8 S. Hayashi, M. Tanaka, H. Hayashi, S. Eu, T. Umeyama, Y. Matano, Y. Araki and H. Imahori, J. Phys. Chem. C, 2008, 112, 15576-15585.

9 P. Shen, Y. Liu, X. Huang, B. Zhao, N. Xiang, J. Fei, L. Liu, X. Wang, H. Huang and S. T. Tan, Dyes Pigm., 2009, 83, 187-197.

10 D. Liu, B. Zhao, P. Shen, H. Huang, L. Liu and S. T. Tan, Sci. China, Ser. B: Chem., 2009, 52, 1198-1209.

11 F. Sanda, T. Nakai, N. Kobayashi and T. Masuda, Macromolecules, 2004, 37, 2703-2708.

12 (a) V. Promarak, A. Punkvuang, T. Sudyoadsuk, S. Jungsuttiwong, S. Saengsuwan, T. Keawin and K. Sirithip, Tetrahedron, 2007, 63, 8881-8890; (b) S. Jagadeeswari, G. Paramaguru, S. Thennarasu and R. Renganathan, J. Mol. Struct., 2014, 1060, 191-196.

13 Z. S. Wang, N. Koumura, Y. Cui, M. Takahashi, H. Sekiguchi, A. Mori, T. Kubo, A. Furube and K. Hara, Chem. Mater., 2008, 20, 3993-4003.

14 S. Cai, X. Hu, J. Han, Z. Zhang, X. Li, C. Wang and J. Su, Tetrahedron, 2013, 69, 1970-1977.

15 M. Liangwab and J. Chen, Chem. Soc. Rev., 2013, 42, 34533488.

16 (a) M. D. Zhang, H. X. Xie, X. H. Ju, L. Qin, Q. X. Yang, H. G. Zheng and X. F. Zhou, Phys. Chem. Chem. Phys., 2013, 15, 634-641; (b) T. Sudyoadsuk, S. Pansay, S. Morada, R. Rattanawan, S. Namuangruk, T. Kaewin, S. Jungsuttiwong and V. Promarak, Eur. J. Org. Chem., 2013, 5051-5063; (c) N. Koumura, Z. S. Wang, M. Miyashita, Y. Uemura, H. Sekiguchi, Y. Cui, A. Mori, 
S. Mori and K. Hara, J. Mater. Chem., 2009, 19, 4829-4836; (d) S. Cai, G. Tian, X. Li, J. Su and H. Tian, J. Mater. Chem. A, 2013, 1, 11295-11305; (e) A. Venkateswararao, K. R. Justin Thomas, C.-T. Li and K.-C. Ho, RSC Adv., 2015, 5, 1795317966.

17 H. Tian, X. C. Yang, R. K. Chen, R. Zhang, A. Hagfeldt and L. Sun, J. Phys. Chem. C, 2008, 112, 11023-11033.

18 B. C. O'Regan and F. J. Lenzmann, J. Phys. Chem. B, 2004, 108, 4342.

19 P. Gao, Y. J. Kim, J. H. Yum, T. W. Holcombe, M. K. Nazeeruddin and M. Grätzel, J. Mater. Chem. A, 2013, 1, 5535-5544.

20 W. H. Nguyen, C. D. Bailie, J. Burschka, T. Moehl, M. Grätzel, M. D. McGehee and A. Sellinger, Chem. Mater., 2013, 25, 1519-1525.

21 (a) K. R. J. Thomas, Y. C. Hsu, J. T. Lin, K. M. Lee, K. C. Ho, C. H. Lai, Y. M. Cheng and P. T. Chou, Chem. Mater., 2008, 20, 1830-1840; (b) J. Song, F. Zhang, C. Li, W. Liu, B. Li, Y. Huang and Z. Bo, J. Phys. Chem. C, 2009, 113, 1339113397; (c) P. G. Hoertz, R. A. Carlisle and G. J. Meyer, Nano Lett., 2003, 3, 325-330; (d) G. Paramaguru, R. Vijay Solomon, S. Jagadeeswari, P. Venuvanalingam and R. Renganathan, J. Photochem. Photobiol., A, 2013, 271, 31-
44; (e) G. Paramaguru, R. Vijay Solomon, S. Jagadeeswari, P. Venuvanalinga and R. Renganathan, Eur. J. Org. Chem., 2014, 753-766; (f) G. Paramaguru, A. Chandiran, P. Gao, R. Rajalingam, M. Grätzel and Md. K. Nazeeruddin, J. Phys. Chem. C, 2014, 118, 16896-16903.

22 M. K. Nazeeruddin, A. K. Rodicio, R. Humpbry-Baker, E. Müller, P. Liska, N. Vlachopoulos and M. Grätzel, J. Am. Chem. Soc., 1993, 115, 6382-6390.

23 (a) A. Baheti, P. Singh, C. P. Lee, K. R. Justin Thomas and K. C. Ho, J. Org. Chem., 2011, 76, 4910-4920; (b) G. Paramaguru, A. Chandiran, P. Gao, R. Rajalingam, M. Grätzel and Md. K. Nazeeruddin, ChemPhysChem, 2015, 16, 1035-1041.

24 Y. Ooyama, Y. Hagiwara, Y. Oda, T. Mizumo, Y. Harima and J. Ohshita, New J. Chem., 2013, 37, 2336-2340.

25 (a) P. B. Pati and S. S. Zade, Tetrahedron, 2013, 69, 21672174; (b) S. Jagadeeswari, G. Paramaguru, R. Madhumitha, Md. K. Nazeeruddin and R. Renganathan, J. Photochem. Photobiol., A, 2015, 299, 194-202.

26 L. M. Peter, Phys. Chem. Chem. Phys., 2007, 9, 2630-2642.

27 B. C. O'Regan and J. R. Durrant, Acc. Chem. Res., 2009, 42, 1799-1808. 